\title{
High Temperature Interface Superconductivity
}

\author{
A. Gozar ${ }^{a}$ and I. Bozovic ${ }^{a, b}$ \\ ${ }^{a}$ Yale University, New Haven, CT 06511, USA \\ ${ }^{b}$ Brookhaven National Laboratory, Upton, NY 11973, USA
}

\begin{abstract}
High- $\mathrm{T}_{\mathrm{c}}$ superconductivity at interfaces has a history of more than a couple of decades. In this review we focus our attention on copper-oxide based heterostructures and multi-layers. We first discuss the technique, atomic layer-by-layer molecular beam epitaxy (ALL-MBE) engineering, that enabled High- $\mathrm{T}_{\mathrm{c}}$ Interface Superconductivity (HT-IS), and the challenges associated with the realization of high quality interfaces. Then we turn our attention to the experiments which shed light on the structure and properties of interfacial layers, allowing comparison to those of single-phase films and bulk crystals. Both 'passive' hetero-structures as well as surface-induced effects by external gating are discussed. We conclude by comparing HT-IS in cuprates and in other classes of materials, especially Fe-based superconductors, and by examining the grand challenges currently laying ahead for the field.
\end{abstract}

\section{Introduction}

Interfaces between different materials can have quantitatively modified properties compared to the bulk of the constituents, and even display qualitatively new phenomena. This can happen for several reasons and perhaps the foremost is expected to be structural. Symmetry at an interface is also different than in the translationally invariant bulk. As a result, artificial and metastable phases of matter, the so called "interface compounds", can become energetically favorable so as to ensure a smooth layering sequence in terms of stoichiometry and bonding, see Fig.1 [1]. Another reason are the proximity effects: the physical properties in near-interface region of one of the constituent materials may be modified due to the influence of the other constituent. This can be especially interesting for complex oxides which display a 
plethora of functional properties like magnetism, ferro-electricity or superconductivity. Combining them in a single interface compound can be useful for a variety of applications. Last but not least, the dimensionality of the surfaces and interfaces is lower, and hence on would expect that correlations, screening, disorder or fluctuations can play a more important role in the electronic dynamics. In conjunction with surface localized states and excitations, these can lead to novel phenomena.

In terms of superconductivity, the sign and the strength of pairing interactions at surface can be different than in the bulk. Half a century ago Ginzburg proposed that in an otherwise insulating material a near-surface layer can acquire metallic character and even superconduct if surface states are partially filled [2]. Such ideas have found experimental validation in the last few decades.

So far two main routes have been investigated in order to produce highquality interfaces. One is hetero-epitaxy which implies the growth of two or more compounds on commercially available substrates. Multi-layers made out of copper-oxide based high temperature superconductors (HTS) or those made of band or Mott-type insulating oxides with perovskite-like structures, e.g. $\mathrm{LaAlO}_{3} / \mathrm{SrTiO}_{3}(\mathrm{LAO} / \mathrm{STO})$ are among the most prominent realizations in the field [3-6]. A second method is to intervene post-growth and change the properties of a surface or interface layer by applying an external agent, for example by exposure to a chemically reactive environment or by applying a voltage bias to the hetero-structure.

\section{ALL-MBE: the technology behind HT-IS}

However, as natural occurrences of perfect and useful interfaces in bulk crystalline solids are scarce, they have to be artificially engineered. This process requires state-of-the-art synthesis and control capabilities.

Atomic layer-by-layer molecular beam epitaxy (ALL-MBE) has been used for nearly three decades for fabrication of HTS films, multi-layers and devices $[7,8]$. The technique allows for precise control of the growth conditions and of atomic fluxes aimed at the substrates, down to about one percent of monolayer coverage. The main part of the oxide ALL-MBE system at Brookhaven, see Fig. 2, is an ultra-high vacuum (UHV) chamber $\left(\mathrm{p} \leq 10^{-9}\right.$ Torr) which hosts sixteen elemental sources along with characterization tools. Atomic species are evaporated from thermal effusion cells at a shallow 
angle $\left(\theta=20^{\circ}\right)$ with respect to the substrate. On one hand this allows for insertion of additional real-time characterization devices, e.g. ion scattering or low energy electron spectroscopy instrumentation but, more importantly, it enables achieving a finite gradient in the spread of atomic concentration at the substrate level. This is crucial for growth of combinatorial samples with unprecedented fine separations in composition, a topic which will be discussed in more detail later in this review.

A brief description of the instrumentation for growth monitoring in the main chamber is as follows (see also Fig. 3). Accurate stoichiometry is ensured by parallel automatic control of the sixteen pneumatic source shutters in conjunction with flux monitoring tools. The deposition rates are calibrated before growth by a quartz crystal monitor simultaneously with the 16-channel (one for each source) parallel atomic absorption spectroscopy setup. The latter is then used for real time flux monitoring during growth. Each source has its own turbo pumping station and is separated by a gate valve from the main chamber. This configuration reduces the down-time of the system considerably because it is possible to replace/replenish each source independently without breaking UHV in the main growth chamber. Samples are grown in a mixture of $\mathrm{O}_{2}: \mathrm{O}_{3}$ that is distilled by condensation, delivering pure ozone to the film surface. The sample manipulator can host a 3" wafer onto which one can mount up to a $4 \times 4$ array of $1 \mathrm{~cm}^{2}$ substrates. Heating is realized by four individually adjustable quartz lamps and the temperature of the wafer is controlled by four thermocouples and measured by a black-body infrared pyrometer. The crystal quality of the growing film is monitored in real time by reflection high-energy electron diffraction (RHEED).

The main MBE chamber is connected by a UHV transfer tube to a processing chamber so that samples can be transferred between them without being exposed to air. The processing chamber is placed into a class 1000 clean room and has a few in-situ device fabrication capabilities: electronbeam evaporation of various materials used for insulation or metallization, Ar-ion milling and ozone plasma treatments. The clean room hosts an additional battery of device fabrication and characterization utilities: profilometry, micro-controlled probe station for room temperature transport, mask aligner for UV-lithography, and a wire bonder. The clean room capabilities of the laboratory are accompanied by additional characterization setups like hard X-ray diffraction (XRD), dedicated vacuum and ozone annealing chamber and atomic force microscopy (AFM). These characterization and fabrication capabilities are further extended by a few advanced research 
tools: $0.3 \mathrm{~K}-300 \mathrm{~K}$ range DC transport for combinatorial and Hall measurements in liquid $\mathrm{He} 4$ and $\mathrm{He} 3$, as well as in extremely temperature-stabilized closed-cycle cryocooler setups [9]. We also use state-of-the-art custom-made $\mathrm{AC}$ mutual inductance and sheet conductance setups for susceptibility measurements in the superconducting state.

\section{HT-IS: history and development}

In this section we review early results as well as data up to about a few years ago on HT-IS. Additional information can be found in several previously published reviews $[4,10]$. The first results on HT-IS in cuprates were obtained by J. Eckstein and I. Bozovic at Varian Research in California [7] only a few years after the discovery of HTS materials [11]. The structure shown in Fig. 4 was made by virtue of the ALL-MBE technique and it also represents the first realization of an artificial superconductor. The metastable $\mathrm{Bi}-22(\mathrm{n})(\mathrm{n}+1)$ compound is realized by insertion of $\mathrm{n} \times\left(\mathrm{CaCuO}_{2}\right)$ layers in the basic structure of $\mathrm{Bi}-2201\left(\mathrm{Bi}_{2} \mathrm{Sr}_{2} \mathrm{CuO}_{6}\right)$. Fig. 4 shows an example of a structure containing a single layer of $\mathrm{Bi}-2278(\mathrm{n}=7)$. These results confirmed that HTS is essentially a 2D phenomenon and also provided the first hints of unusual proximity effects in these materials.

The year 2002 marked the first report of an interfacial-induced $T_{c}$ enhancement in hetero-structures based on $\mathrm{La}_{2-\mathrm{x}} \mathrm{Sr}_{\mathrm{x}} \mathrm{CuO}_{4+\delta}$ (LSCO) family of compounds [12]. A bilayer system consisting of a 5 UC oxygen doped superconducting LCO materials grown on top of a 15 UC optimally doped superconducting LSCO, $\mathrm{x}(\mathrm{Sr})=0.15$, showed $T_{c} \approx 51.5 \mathrm{~K}$. This represented a dramatic increase in $T_{c}$ because hundreds of single-phase films grown under similar conditions showed, without exception, a maximum $T_{c} \approx 40 \mathrm{~K}$. While much of what is going on in this type of heterostructures has been clarified in the meantime, pinning down the origin of the interfacial enhancement remains an outstanding challenge.

Our understanding of HT-IS advanced dramatically in the 2007 - 2010 period by virtue of a series of detailed studies of heterostructures like the one shown in Fig. 6. The superconducting dome in LSCO compounds as a function of $\mathrm{Sr}$ concentration starts around $\mathrm{x}(\mathrm{Sr}) \approx 0.06$, it reaches a maximum $T_{c}$ of about $40 \mathrm{~K}$ at the optimal $(\mathrm{OP})$ value $\mathrm{x}(\mathrm{Sr}) \approx 0.16$ and dies out for $\mathrm{x} \approx$ $0.25-0.3$, following a conjectured parabolic shape. The basic building blocks of several studies are bilayers with $\mathrm{x}(\mathrm{Sr})=0$ (denoted by $\mathrm{I}$, insulator) and $\mathrm{x}(\mathrm{Sr})=0.45$ (denoted by $\mathrm{M}$, metal) in which the constituents are on either 
side of the SC dome but not superconducting per se [3]. If LCO is annealed in ozone it becomes superconducting itself and it is denoted by S. Fig. 6 shows a M-I bilayer (the $1^{\text {st }}$ letter denotes the material closest to the substrate). Our data showed that I-M bilayers have $T_{c} \approx 15 \mathrm{~K}$, M-I structures have $T_{c} \approx 30 \mathrm{~K}$ and M-S bilayers have enhanced $T_{c} \approx 51 \mathrm{~K}$ [3], as obtained by resistance and mutual inductance measurements which were first acquired on relatively large $\left(1 \times 1 \mathrm{~cm}^{2}\right.$ area $)$ films.

The first information about the thickness of the interface layer was obtained by synthesis of a series of I-M and M-I samples with thick ( 30 UC) bottom layers while the thickness of the top layer was increased digitally, one formula unit at the time. Plateau values for $T_{c}$ were reached very soon, after depositing only about 2 unit cells (UC) of the top layer (a UC in LSCO contains two formula units). This is shown in Fig. 7. These data already indicated that the HTS layer must be very thin because the top layers contained very few $\mathrm{CuO}_{2}$ planes.

One of the obvious questions prompted by these results was how thick was actually the interface layer with the highest $T_{c}$. Transport and superfluid density measurements in a battery of such samples where each one of them had a specific layer doped with Zn, see Fig. 8, addressed this challenging question in a straightforward and spectacularly successful way [13]. They showed that HT-IS is confined to a single $\mathrm{CuO}_{2}$ plane. The latter studies took advantage of the known fact that $\mathrm{Zn}$ suppresses superconductivity very efficiently [14,15]: only 3\% doping already reduces $T_{c}$ in optimally doped LSCO by a factor of about two and this dramatic effects appear to be approximately proportional across the phase diagram [15]. These data allowed us to pinpoint the exact location of the HTS plane with atomic resolution.

We also addressed two outstanding issues related to HT-IC in these bilayers. The first was related to the mechanism by which the layer with the highest $T_{c}$ gets doped: whether it was due to pure stoichiometry, i.e. $\mathrm{La} / \mathrm{Sr}$ interdiffusion, or to charge transfer, or both. Previous c-axis transport studies in LSCO-based multilayers [16] indicated clearly that inter-diffusion, if present, must take place on a very small scale: 1 UC of LCO sandwiched between OP-LSCO stayed insulating while 1 UC of OP-LSCO retained its highest $T_{c}$ when grown between insulating LCO layers. Nevertheless, the question of the stoichiometry profile at the interface in M-I bilayers was addressed directly by two independent techniques: high-resolution crosssection Transmission Electron Microscopy (HR-TEM) and in-situ Time-ofFlight Ion Scattering and Recoil Spectroscopy (TOF-ISARS) [3]. Both of 
these techniques were in quantitative agreement with each other and showed that cation inter-mixing is limited to at most one UC from the nominal interface. Striking evidence for this statement can be seen in Fig. 9.

Furthermore, these results were fully confirmed by several other characterization and transport experiments. Real-time RHEED oscillations showed abrupt changes in intensity, i.e. over one ML length-scale, when crossing from $\mathrm{M}$ to I during the growth of a M-I bilayer. Transport in I-M-I tri-layers showed a maximum $\mathrm{T}_{\mathrm{c}} \approx 15 \mathrm{~K}$, similar to the data in I-M structures instead of $\mathrm{T}_{\mathrm{c}} \approx 30 \mathrm{~K}$ as in M-I bilayers. As the only difference between I-M and I$\mathrm{M}-\mathrm{I}$ structures is the nature of the bottom layer and proximity to the LSAO substrate, these results simply cannot be explained by cation mixing or Oxygen non-stoichiometry at the interface.

While TEM is generally the "go to" technique when it comes to interface structure and morphology analysis, Fig. 9 shows how TOF-ISARS can be used extremely efficiently for the same purpose. One should fully appreciate the power of this surface sensitive technique considering that TOF-ISARS can be applied in-situ during the actual growth, that no further sample preparation is necessary and that minimal analysis of the raw data is needed to extract quantitative profile distribution and sometimes even surface crystallographic information [17]. Given the derived cation profile one can estimate the charge distribution across the interface by solving the Poisson equation for the discrete case of quasi-2 $\mathrm{D} \mathrm{CuO}_{2}$ planes immersed in an effective medium. The results obtained depend only marginally on the fine details of the phenomenological functional form chosen for the chemical potential $\mu=$ $\mu(x)$ where $x$ is the hole concentration and they are fully consistent with the experiment: the $2^{\text {nd }}$ layer from the nominal interface is the "hot" $\mathrm{CuO}_{2}$ plane, i.e. the one with the highest $T_{c}$. Subsequent studies and techniques further confirmed these results quantitatively. The entire body of evidence accumulated so far has established a very solid foundation for these phenomena.

The second problem was to explore the nature of the $25 \%$ enhancement in the M-S structures. Using a two-coil mutual inductance setup in the transmission geometry we were able to show that the enhanced $T_{c}$ is confined in a 1-2 UC thick layer, which we believe must be located at the interface [3]. The thickness of this layer was estimated from non-invasive critical current $\left(I_{c}\right)$ measurements as a function of temperature. A linear $I_{c}(T)$ dependence is expected and has been observed experimentally in high quality films and bulk single-crystals due to vortex depinning or vortex-antivortex pair break- 
ing. However, for M-S bilayers the temperature dependence of the critical current shows a clear break and allows for a straightforward decomposition in two approximately linear regions. The $25 \%$ interface-confined enhancement of $T_{c}$ was inferred from the relative slopes of these two regions.

These findings pose some very intriguing questions about the nature of mechanism responsible for the enhancement, its robustness and its confinement to an ultra-thin interfacial layer. One possible explanation is the "doping without disorder" scenario. This is rooted in two facts, (i) that within of the family copper-based HTS compounds $T_{c}$ can change from $10 \mathrm{~K}$ to about $135 \mathrm{~K}$ which shows that the layers surrounding the $\mathrm{CuO}_{2}$ planes are crucially important for achieving high $T_{c}$ [18], and (ii) that disorder, chemical (e.g., doping with $\mathrm{Zn}$ ) or structural (e.g., oxygen disorder within $\mathrm{CuO}$ chain layers in YBCO), reduces $T_{c}$. Other conceivable explanations may invoke structural differences, suppression of competing orders or a particular oxygen distribution at the interface. The last aspect may be particularly important because enhancement in $T_{c}$ is observed only in ozone-annealed samples. Last but not least, a theory was also put forward that invokes favorable synergy between the large phase stiffness in $\mathrm{M}$ and strong pairing in I layers [19,20].

To address and clarify the interface structure and properties, extensive work has been invested, employing some sophisticated techniques. Substantial progress has been achieved, and we review it briefly in what follows.

Resonant soft X-ray study of a superlattice made out of undoped LCO and metallic, non-superconducting LSCO provide evidence for minimal cation inter-diffusion across the interface, taking place over only about one-half the unit cell height $(0.66 \mathrm{~nm})$ [21]. It also showed electronic redistribution across the interface, over a comparable length scale. Using X-ray energies in the proximity of the $\mathrm{O} \mathrm{K}$ edge to resonantly enhance the sensitivity to the mobile hole distribution, it was found that the profile of carriers is different (smoother and spread out more) from that of the $\mathrm{Sr}^{2+}$ cations, demonstrating true electronic effect (charge accumulation on one side of the geometric interface and depletion on the other). These results are in quantitative agreement with the bilayer data in that the separate contributions of interface roughness and that of charge transfer are clearly measurable and have similar magnitude, as illustrated in Fig. 11a.

Studies of LSCO M-I bilayers by Coherent Bragg Rod Analysis (COBRA) method revealed an interesting structural effect - large changes in $c_{A}$, the distance between a copper atom and the nearest apical-oxygen atom. CO- 
BRA is a synchrotron-base X-ray scattering technique especially suitable for ultra-thin films. It uses efficient phase-retrieval algorithms in order to analyze diffracted intensity along entire non-equivalent Bragg rods [21]. The main result of this study was to show that, as opposed to single-phase films, there is a dramatic increase in $c_{A}$, by as much as $0.45 \AA$ as one goes from the M-I interface towards the bilayer film surface, see Fig. 11b. This result is important in that empirically it was found that $T_{c}$ in cuprates scales with $c_{A}$, (higher $T_{c}$ corresponds to longer $c_{A}$ ), and these correlation was proposed by some to carry deeper physical meaning and play a crucial role for HTS.

These results, which show unexpected behavior rooted to the presence of MI interfaces, have been naturally tied to long ranged effects of the Coulomb interactions in HTS materials because most of the crystal cohesion energy comes from the Madelung terms. This is especially important in the underdoped region where screening is poor and such interactions are expected to be long-ranged. Butko et al. [23] demonstrated this aspect in a straightforward way by systematic X-ray measurements of the c-axis lattice constants in M-I and I-M bilayers, in comparison with those in single-phase $\mathrm{M}$ and I films. In bulk LSCO crystals, as the Sr dopant concentration is increased, the out-of-plane lattice constant increases while the in-plane one decrease. This trend is qualitatively preserved in ALL-MBE grown films. The main finding of Butko et al. was that each bilayer shows only a single value of the out-of-plane lattice constant with no splitting discernible within the experimental resolution. At the same time, the in-plane lattice constants of the top layer were found in each case to be equal to those of the bottom layers, so the top layer is under full epitaxial constraint.

Straightforward conclusions from these finding are as follows. First, the critical thickness of I layer is very different if grown on $\mathrm{M}$ buffer or on the insulating LSAO substrate. Second, the magnitude of the effect reported here cannot be fully accounted for by the epitaxial constraints along with the values of elastic constants as derived for the bulk crystals. This is a direct evidence of the long-ranged 'Madelung strain'. This brings to the forefront the importance of the nature of the substrate layers and sheds light on the nature of the asymmetry in $T_{c}$ between M-I, I-M and the puzzling results obtained in I-M-I tri-layers [3].

Another striking observation of this study is the almost perfect linear scaling of $T_{c}$ with the value of the c-axis lattice constant in LSCO-based films and heterostructures, see Fig. 12. Although very compelling, the interface enhancement still retains part of its mystery. If $T_{c}$ enhancement originated 
simply in the expansion of the crystal structure along the c-axis, one would expect that in the M-S structure the entire top $\mathrm{S}$ layer would have the same $T_{c} \approx 51 \mathrm{~K}$, because all unit cells have the same c-axis lattice constant. However, we know that this is an effect confined only to a very thin layer, at most 1 UC thick, near the interface.

\section{HT-IS with electrolyte doping}

In heterostructures as described above the properties of the interfacial layer at the end of the synthesis stage are determined by the layering sequence. New possibilities open if one is able to subsequently tune the properties of such layers by some external agent. In particular, changing the carrier density in a Field-Effect-Transistor (FET) type of devices had been a longstanding goal. The basic difficulty stems from achieving the electric fields high enough to produce a detectable change in the carrier density. Order-ofmagnitude estimates can be obtained by using the Thomas-Fermi approximation for $\lambda_{T F}$, the screening length: $\lambda_{T F} \propto n^{-1 / 6}$, where $\mathrm{n}$ is the 3D carrier density. For $n \sim 10^{23} \mathrm{~cm}^{-3}$ like in a typical metal, $\lambda_{T F} \sim 0.5 \AA$. In optimally doped HTS cuprates the 3D carrier density is of the order of $n_{3 D} \sim 10^{21} \mathrm{~cm}^{-3}$ and the corresponding $\lambda_{T F} \sim 1 \AA$. So, an external electric field could cause significant charge depletion or accumulation only within a layer less than 1 $\mathrm{nm}$ thick. This in turn necessitates a state-of-the-art film quality in terms of surface smoothness, crystallinity, and stoichiometry. The second grand challenge is related to the magnitude of the external (polarization) charge that need to be brought to the proximity of the HTS film - it should be comparable to the effective sheet density in cuprates at optimal doping which in LSCO is $n_{2 D} \sim 10^{14} \mathrm{~cm}^{-2}$. Such surface polarization values could be induced by fields of the order of $10^{8} \mathrm{~V} / \mathrm{cm}$, a value which is however about one order magnitude higher than the highest achieved breakdown fields in known oxide gate insulators. In LTS materials the charge carrier densities are even higher, making the requirements to substantially modify the surface layer are even more stringent [24].

Ferroelectric gating was used with success to dynamically change the carrier densities in superconducting FET structures. In such devices, the state of a ferroelectric like $\mathrm{PbZrTiO}$ is switched by an applied gate voltage. This leads to 'digital' changes in the near-interface carrier density induced by the switching of polarization charges. Shifts in $T_{c}$ by several degrees were obtained for example in $\mathrm{GdBa}_{2} \mathrm{Cu}_{3} \mathrm{O}_{7-\delta}$ or $\mathrm{NdBa}_{2} \mathrm{Cu}_{3} \mathrm{O}_{7-\delta}$ films $[25,26]$. 
A breakthrough in this field was achieved when the typical solid gate dielectric was replaced by an ionic liquid or a polymer electrolyte, see Fig. 13. when a voltage is applied between the film and the gate electrode, mobile ionic charges accumulate near their surfaces. In addition to a high film surface quality, the new challenge for the realization of the ideal electric double-layer transistor device (EDLT) is chemical compatibility between the oxide film and the electrolyte. No chemical reactions should take place between the film and the electrolyte, and the interface should be impermeable to the passage of ions from one side to the other. Pioneering work in the field of HTS electrolyte FET devices was carried out more than two decades ago by McDevitt and collaborators [27-31]. Reversible changes of $T_{c}$ were induced in $\mathrm{YBaCuO}, \mathrm{BSCCO}$, and TBCCO films, as high as $\Delta T_{c}>50 \mathrm{~K}$. The same group also successfully produced home-made electrolytes that remained liquid down to low temperatures, allowing them to modify the properties of the films while in superconducting state.

This technique was revived about 15 years later in works involving various oxides like $\mathrm{ZnO}, \mathrm{NiO}$ or $\mathrm{SrTiO}_{3}$ [32-34]. The results showed that the doping range achieved by this technique can largely exceed what had been previously realized in conventional FET setups. Since then electrolyte-FET has been applied to many other materials including various complex oxides. Among the remarkable achievements is the discovery of $\mathrm{SC}$ in $\mathrm{KTaO}_{3}$, which was not achievable by chemical doping [35]. In most of these papers, an ideal FET picture was used as the standard framework in which the data were analyzed. However, the validity of this picture came under closer scrutiny and it was questioned in several instances at least [36-38]. New techniques have been developed to test how the actual doping mechanism in EDLT devices, and in fact recent data indicate that this mechanism varies depending on the chemical compatibility of the electrolyte-oxide pair used in experiment. We believe that in the near future such tests will become standard for the field.

In the following we focus on how this technique was used to successfully tune the carrier density over a wide range in HTS cuprates, see also Fig. 13. Using a LSCO-based hetero-structure grown by ALL-MBE, Bollinger et al. tuned the sample over the superconducting-insulator transition achieving shifts in $T_{c}$ by up to $30 \mathrm{~K}$ [39]. Unless a characteristic threshold voltage was exceeded the measurements were reversible and reproducible. Moreover, gating worked in both directions: both electron accumulation or depletion were achieved. Hundreds of curves corresponding to temperature dependent sheet resistance at various gate voltages, $R\left(T, V_{G}\right)$ were recorded and they all 
could be collapsed on a single universal function as predicted for a $2 \mathrm{D}$ superconductor-insulator transition. The observed critical resistance was found to be precisely $R_{Q}=h /(2 e)^{2}=6.45 \mathrm{k} \Omega$, i.e., the quantum resistance for pairs. Almost simultaneously, very similar results were reported for the $\mathrm{YBaCuO}$ system by the group of Allen Goldman [40]. Very good scaling of the resistance was obtained in the $6 \mathrm{~K}-22 \mathrm{~K}$ temperature range. They inferred a slightly smaller value of the critical resistance, $R_{c}=6 \mathrm{k} \Omega$. Note that in previous studies of insulator-superconducting transitions in other oxide or in disordered conventional metals, the obtained value of the critical resistance was not universal $[41,42]$. The very good agreement with the $2 \mathrm{D}$ theory obtained in the work on LSCO structures [39] attests to capability of ALLMBE to fabricate extremely thin active layers, where the effects are likely to be confined to a single $\mathrm{CuO}_{2}$ plane, while maintaining a high film quality.

\section{HT-IS in cuprates: recent progress}

The body of work discussed above has established the physics of complex oxide interfaces, and in particular that of HT-IS, as important milestone on the current roadmap leading to exploiting the functional behavior of these materials and perhaps even achieving the holy grail of room temperature SC. More recent studies, which are the focus of this section, have brought new understanding and showed that even LSCO based HT-IS, although already well studied, have not run out of surprises.

We discussed before the importance of the ionic component in the binding energy of HTS materials and its connection to the long-ranged Madelung strain [23]. A recent COBRA study, augmented by a novel energydifferential method in order to determine the concentration of specific atoms in shared lattice sites, uncovered a new result in ultra-thin LSCO films with $\mathrm{x}(\mathrm{Sr})=0.44$ [43]. It is known that one formula unit in LSCO consists of one negatively charged $\mathrm{CuO}_{2}$ layer 'sandwiched' between two compensating $\mathrm{La} / \mathrm{SrO}$ cation layers. One finding of this work is that the sequence of $\mathrm{La} / \mathrm{Sr}$ and $\mathrm{Cu}$ layers always follow the $\mathrm{La} / \mathrm{SrO}-\mathrm{CuO}_{2}-\mathrm{La} / \mathrm{SrO}$ progression independent of the shuttering sequence. Perhaps the simplest and most straightforward way to interpret this result is by invoking the polarization catastrophe: a different layering sequence would induce in LSCO an unsustainable internal voltage, similar to what has been discussed in connection to LAO/STO heterostructures (see Fig. 14a,b). However, the main and most surprising result of this study was that the Sr dopant concentration is systematically much lower in monolayers just below the $\mathrm{CuO}_{2}$ planes and way 
above the average in the layer just above it, see Fig. 14. One could imagine, in a very simplified picture, that the expected $\mathrm{La}_{0.78} \mathrm{Sr}_{0.22}-\mathrm{CuO}_{2}-\mathrm{La}_{0.78} \mathrm{Sr}_{0.22}$ sequence is changed to $\mathrm{LaO}-\mathrm{CuO}_{2}-\mathrm{La}_{0.56} \mathrm{Sr}_{0.44}$. The inversion symmetry is broken and the result effectively implies the existence of a finite dipole moment, with the fixed orientation, in ultra-thin overdoped LSCO. Notably, this is not due to the typical soft-mode condensation mechanism as in ferroelectrics but because of the frozen-in cation redistribution. The finite dipole further implies that the voltage divergence showed in Fig. 14a is at work in this case.

It would be interesting to check whether there is a critical thickness associated with this non-symmetric Sr distribution, as well as whether it can be changed post-growth, for example by the use of piezoelectric or electricforce microscopy at elevated temperatures. It would be also interesting to study these films by TOF-ISARS in-situ and check if the non-symmetric $\mathrm{Sr}$ distribution takes place in real time or in already buried layers. The magnitude of the reported effect is substantial and with potential of further enhancement, which could affect in a non-trivial way fundamental superconducting properties, e.g., the symmetry of the order parameter. The physical basis for such results may largely reside in the way the electrostatic energy of the entire structure (substrate + film + vacuum interface) is balanced out during growth. A phenomenology similar in spirit to that employed successfully for the understanding of the retention of the polarization state in ultrathin ferroelectric films as a function of the external electrode nature and configuration could be also useful in this case [44-46]. Boundary conditions play a crucial role in the stabilization of a given internal structure, which is just another clear manifestation of the 'Madelung strain'.

The problem of interface superconductivity and the nature of $T_{c}$ enhancement was tackled recently in a combinatorial study using an unprecedentedly large sample set [47]. HT-IS was studied in a library of M-I bilayer samples in which the doping of the bottom, M, layer was changed from $0.15<\mathrm{x}(\mathrm{Sr})$ $<0.47$, in extremely fine steps. This work took advantage of the concentration gradient in the molecular beam due to the shallow angle made by the thermal evaporation sources and the substrate plane. This allows for an essentially continuous tuning of the chemical composition. Combinatorial libraries of samples were lithographically patterned and measured in a specially designed high throughput parallel setup, see Fig. 15. The key result of the paper is that across the entire doping range studied the ALL-MBE grown LSCO films showed remarkable uniformity, less than $1 \%$ variation in the 
measured superconducting $T_{c}$. It is important to note that while there is some uncertainty in defining $T_{c}$ from $\mathrm{R}(\mathrm{T})$ curves, the films show hardly any variation if the same criterion is consistently applied. The experimental results were compared to what was expected based on the well-documented contributions of charge transfer and cation redistribution at the M-I interface combined with a phenomenological parabolic $T_{c}(x)$ dependence, which is widely assumed to hold for bulk LSCO samples, as well as with a putatively linear dependence of the chemical potential $\mu(\mathrm{x})$ originating from a cylindrical Fermi surface. The absence of any $T_{c}$ variation is not consistent with this model and constrains the variations of the chemical potential with the doping level. This fact was already demonstrated by the lack of charge redistribution across the interfaces in LCO-LSCO tri-layers [16]. Last but not least, this work addresses the notion of possible interface enhancement due to phase fluctuations in the underdoped LSCO being quenched by proximity effects to the highly doped metallic LSCO layer. The fact that in M-I bilayers $T_{c}$ is constant independent on doping (across the entire $0.15<\mathrm{x}<0.47$ range) in the metallic layer range does not agree with this theoretical proposal.

The phase diagram of cuprates shows that their parent compounds are antiferromagnetic Mott insulators of the charge transfer type which, if doped, can be turned into Fermi-liquid like systems on the other side of the superconducting dome. It is thus interesting to check how their magnetic and electromagnetic response evolve in ultra-thin layers with carrier concentration. As for the electrodynamics, we only note that a recent study addressed the disputed issue of the $\mathrm{BKT}$ physics in a single $\mathrm{CuO}_{2}$-thick layer, as encountered in M-I bilayers [48]. Measurements of complex sheet conductance employing a single-coil inductance technique operated in the $2-30 \mathrm{MHz}$ range showed a steep jump in the superfluid density at $T_{B K T}$. Generally, it was found that the response of the film can be well described by the BKT theory extended to finite frequencies.

Inelastic X-ray scattering (RIXS) studies of ALL-MBE grown heterostructures addressed another important the issue, the dependence of magnetic correlations on doping and layer thickness. In [49], magnetic excitations in a single UC thick undoped LCO layer were studied by RIXS. The samples consisted of multilayers of 1 and 2 UC's of LCO sandwiched between non-magnetic $\mathrm{LaAlO} 3$ (LAO) layers. The measurements revealed that well-defined magnons - coherent transverse oscillations of the staggered magnetization - persist in LCO layer thickness down to 1 UC level, and 
that their dispersion is almost undistinguishable from the one found in bulk LCO crystals. No evidence was found for more complex correlations, e.g., of the resonating valence bond type, which would modify the dispersion close to the Brillouin zone boundary. Although more work is needed to completely elucidate the nature of the high-energy magnetic continuum, the result that the picture based on classical correlations seems to describe well a genuinely $2 \mathrm{D} S=1 / 2$ spin system is remarkable. In a subsequent RIXS study [50], the evolution of the magnetic correlations with carrier doping was mapped out. The main finding of that work was that across the entire phase diagram, the magnetic excitations persist and have similar intensities and dispersions at high energies as in the undoped LCO case, see Fig. 15; the only significant difference is in some broadening at increased doping. The main implication for the HTS is that the demise of superconductivity on the overdoped side is not caused by the changes in high energy magnetic excitations.

\section{HTIS in Fe-based superconductors}

Until about a decade ago HTS basically meant $\mathrm{Cu}$-based materials. Fe-based systems, also known as pnictides because many of them contain elements from the $5^{\text {th }}$ column of the periodic table like As or $\mathrm{P}$, became in the recent years another important family of HTS compounds. Since the beginning, one of the key questions was whether the Fe-based superconductors can be described within the conventional BCS theory, or they share the same HTS mechanism with the cuprates, or perhaps host something else. Until recently the highest $T_{c} \approx 56 \mathrm{~K}$ was observed in bulk $\mathrm{Sr}_{0.5} \mathrm{Sm}_{0.5} \mathrm{FeAsF}$ but this situation was apparently changed dramatically by a report of superconductivity above $100 \mathrm{~K}$ in a single layer of FeSe grown on specially prepared $\mathrm{SrTiO}_{3}$ (STO) substrates [51]. This achievement followed up on previous reports which hinted that the putative superconducting gaps (as seen by STM or ARPES) persisted to substantially higher temperatures than what was found in bulk FeSe samples [52]. It is to be noted that the high $T_{c}$ reported in [51] was inferred not from standard four-probe resistivity measurements but from the slope of I-V characteristics, measured using a modified STM probe with 4 tips. It is important that this result is further confirmed by magnetic susceptibility measurements in the superconducting state.

One of the problems with FeSe is that it is very air sensitive and this hampered standard transport measurements. It was fund that as soon as the materials is capped with a protective layer, $T_{c}$ drops dramatically. This indicated 
the need for in-situ studies. The technical solution was to integrate the MBE synthesis chamber with other characterization tools so that the sample can be measured without breaking the UHV. In these setups STM and ARPES provided a wealth of important information: it was found that FeSe is doped by electron accumulation, that it showed a circular Fermi surface and that the superconducting gap is isotropic. For more direct experimental evidence, the group of Jin-Feng Jia developed an important technical capability in the form of in-situ four-point contact probe that can measure $R(T)$ in applied fields up to $11 \mathrm{~T}$. The resistance was showed to drop sharply towards zero at $T_{c}=109 \mathrm{~K}$.

Given the difficulty of the experiment and many open questions, independent confirmation of these results would be highly desirable. In particular, the development of in-situ tools for measuring magnetic screening properties, for example a mutual inductance setup, may be crucial for confirming these results. Nevertheless, the importance of this work, if validated, is very high for the HTS community; the spectacular interface enhancement of $T_{c}$ needs an explanation. However, few plausible ideas and candidates explanations like nesting, multi-band superconductivity, the paramount role of hole vs. electron doping, unconventional symmetry of the order parameter, etc., can already all be essentially ruled out. A host of theories proposed that HTS originates in the enhancement of the pairing interaction across the interface. A consensus is however still to be reached in whether this is due to the high value of the dielectric function in STO, or to the strong electron phonon coupling to either soft acoustic branches or to optical excitations in the substrate, or something else yet to be discovered and elucidated.

\section{Conclusions and outlook}

Before delving into too many exotic theories, the observation of HT-IS with $T_{c}>100 \mathrm{~K}$ in FeSe/STO needs to be reproduced and confirmed by other groups and other techniques. Important progress can be achieved by obtaining systematic information about the interface structure, morphology and carrier distribution, in a manner similar to what has been done for cuprates. This highlights the importance of continuing to perfect and develop new and versatile tools for the study of interface physics. We will now briefly discuss this topic along with the grand challenges that lay ahead of the HT-IS field.

This review touched upon a few powerful experimental techniques for the study of interfaces. Perhaps one of the most useful and widely used is based 
on TEM and its variants like STEM, Z-contrast, or high-resolution electron energy-loss spectroscopy (HR-EELS). A wealth of information was brought by COBRA and its recent upgraded capabilities like energy-differential diffraction. This technique allowed unprecedented insight in the atomic distribution on a layer-by-layer basis in ultra-thin films and across interfaces, including that of light atoms like oxygen or in the occupancy of lattice sites which can be shared by different atomic species. We showed that on par with TEM, TOF-ISARS can provide information about atomic profiles or even interface structure. An intriguing question is whether it has the ability to provide the kind information extracted from COBRA studies. ALL-MBE allowed to attack the problem of interface charge distribution from a new perspective: $\delta$-doping tomography was proposed specifically for mapping the superfluid density along the growth direction, and turned out to be spectacularly successful. It would be very useful to apply this entire battery of established analytical tools to FeSe/STO and newly discovered HTS in other materials.

In terms of new directions, one of the most promising paths is clearly the integration of MBE synthesis with other characterization tools, starting with four-point-contact $R(T)$ and magnetic screening (mutual inductance) measurements, and continuing with more sophisticated setups like STM, ARPES, AFM, or HR-EELS. 'On demand' termination layers may be able to bring in surprises even for thoroughly studied materials like HTS cuprates []. AFM, although not one of the main players so far, can be very useful for the study of buried ultrathin films if combined with spectroscopic techniques. In fact, scattering-based Scanning Near Field Microscopy (s-SNOM), which employs AFM as a surface probe and its tip as nano-optical antenna, has been shown to be an effective spectroscopic probe for surface as well as subsurface interfacial layers which are only one atom thick. Besides improved optical spatial resolution which can go orders of magnitude beyond the diffraction limit, light confinement in the proximity of the AFM tip opens the opportunity for an additional degree of freedom not present in typical farfield absorption or Raman setups, i.e., finite momentum optics. It has been shown that s-SNOM can be used for mapping plasmons in the normal and superfluid states. It would be also interesting to make its depth resolution compatible with cross-section scanning as well as to use it for the determination of the thickness of the metallic or insulating layers of interest in conventional or electrolyte-FET setups. 
A lot has been learned about interface superconductivity, much of it using ALL-MBE synthesis and electric field effect (mainly with electrolytes). The array of techniques showed that in the cleanest samples there is a genuine electronic reconstruction, while they also provided evidence for non-trivial atomic displacements. Quasi-2D superconductivity was observed in STO, LSCO, YBCO, etc.; importantly, it was also achieved for the first time in $\mathrm{KaTaO}_{3}$ by electrolyte gating. Some of the interfaces show substantial enhancement in superconducting properties and at this moment each of these cases (LCO/LSCO, FeSe/STO, PbTe/PbS) awaits a more complete understanding. These advancements are useful for marking up the roadmap for future developments and are closely related to the grand challenges laying ahead in this field. A most prominent task is discovering of new HT-IS examples, and in particular the realization of the situation in which none of the constituents are superconducting themselves. An important challenge from the theoretical side is to use existent materials data-base to predict new combinations of materials that will generate IS and HT-IS, predict the corresponding $T_{c}$, as well as to elucidate the mechanisms leading to interfacial $T_{c}$ enhancement.

This work was supported by the Moore Foundation. 


\section{FIGURE CAPTIONS:}

Figure 1. HR-TEM picture of the interface between a $\mathrm{La}_{2-\mathrm{x}} \mathrm{Sr}_{\mathrm{x}} \mathrm{CuO}_{4}$ film grown on a $\mathrm{LaSrAlO}_{4}$ substrate. At the interface the layering sequence corresponds to $1 \mathrm{UC}$ of $\mathrm{La}_{3} \mathrm{AlCuO}_{7}$; this an example of spontaneous formation of an interface compound (from [1]).

Figure 2. The ALL-MBE system at Brookhaven National Laboratory.

Figure 3. Characterization tools. (a) One of the sixteen arms of the MBE chamber showing the effusion cell and associated pumping station and atomic spectroscopy setups. (b) Schematic of the ion scattering spectroscopy setup. (c) RHEED diffraction pattern (left) and the integrated intensity of the specular spot as a function of time during the growth of a $\mathrm{La}_{2} \mathrm{CuO}_{4}$ film (right). Each oscillation corresponds to one formula unit of the material.

Figure 4. HTS film quality. Left: 3D-AFM of a $78 \mathrm{~nm}$ thick LSCO film on LSAO substrate. The surface rms of the over a $50 \times 50 \mu \mathrm{m}_{2}$ area was measured to be $0.3 \mathrm{~nm}$. Right: XRD data of the film on the left displaying finite thickness oscillations in the vicinity of the (004) reflection (from [12]).

Figure 5. A TEM microgram of a tri-layer heterostructure showing a single molecular layer of Bi-2278 sandwiched between two thicker layers of Bi2212 []. Bi-2278 was the first example of an artificial HTS meta-material, synthesized by virtue of atomic-layer engineering (from [7]).

Figure 6. HT-IS with LSCO materials. Left: resistance of the two building blocks of a typical LSCO-based bilayer, each 20 UC thick. (I = LCO, M = LSCO with $\mathrm{x}=0.45)$. Right: a cartoon representing a typical M-I bilayer. 
Figure 7. Dependence of $T_{c}$ on the top layer thickness in I-M, M-I and M-S bilayers. Here, the critical temperature was determined from the midpoint of the resistive transition (from [3]).

Figure 8. Zn doping effects in individual layers. The number $\mathrm{N}$ indicates which $\mathrm{CuO}_{2}$ plane was doped by replacing $3 \%$ of the $\mathrm{Cu}$ atoms by $\mathrm{Zn}$. The notation is same as in Fig. 6 (right). Transport data are shown on the left and statistics on many bilayers on the right (from [13]).

Figure 9. Cation profile at the interface in a M-I bilayer. Left: Raw data representing the normalized decay of the Sr recoil signal on the I side of a M-I bilayer [3]. Right: comparison between the complementary Sr and La distributions at the interface obtained by TOF-ISARS and TEM, respectively.

Figure 10. Interface-induced enhancement in $T_{c}$. Temperature dependence of the "critical" value of the drive coil current, $I_{d}$, for a single-phase $S$ film and a M-S bilayer with the same nominal thickness of the top, S, layer (from [3]).

Figure 11. (a) Soft X-ray results in LCO-LSCO superlattices. The relative individual contributions to the total carrier profile $(p)$ given independently by charge transfer $\left(p^{0}\right)$ and interface roughness $\left(p^{R}\right)$ respectively can be inferred from the derived values of doping in the three inequivalent $\mathrm{CuO}_{2}$ planes in the super-structure: $p_{\max }=0.3, p_{\text {int }}=0.24, p_{\min }=0.18 ; p_{\max }^{0}=0.3$, $p^{0}{ }_{\text {int }}=0.24, p^{0}{ }_{\text {min }}=0.18 ; p^{R}{ }_{\max }=0.3, p^{R}{ }_{\text {int }}=0.24, p^{R}{ }_{\text {min }}=0.18$. (b) Atomic positions derived from COBRA data in M-I bilayers. The $\mathrm{CuO}_{6}$ octahedra (outlined by white lines) are significantly more elongated near the surface (from [20]).

Figure 12. Critical superconducting temperature $\left(T_{c}\right)$ as a function of the caxis lattice constant in LSCO-based single-phase films and heterostructures (from [22]). 
Figure 13. Superconducting-Insulator phase transition induced by electrolyte gating. Left: Typical configuration in an electrolyte double-layer transistor device. Middle: Temperature dependence of the resistance normalized to the quantum value for an initially strongly underdoped film. Right: Scaling of the same parameter with respect to $\left|x-x_{c}\right| \cdot T^{-1 / z y}$. Data from Ref. [38].

Figure 14. (a): Electric field and potential variation from the substrate surface for two layering sequences in ALL-MBE growth. One of them is seen to lead to the so-called polarization catastrophe. (b) Sr concentration (green bars) in $6 \mathrm{UC}$ thick films of metallic LSCO with $\mathrm{x}(\mathrm{Sr})=0.44$, on LSAO substrate. The positions of $\mathrm{CuO}_{2}$ planes are shown in red; a clear asymmetry between top and bottom Sr distribution can be noticed (from [42]).

Figure 15. Absence of variation in $T_{c}$ as the doping level is varied in M layers within combinatorial M-I heterostructures. (a) Temperature dependence of the resistivity of different pixels in the same film. (b) $T_{c}$ as a function of the nominal $\mathrm{Sr}$ concentration (from [46]).

Figure 16. Magnetic scattering in ultra-thin LSCO films. (a) Peak energy dispersion in superlattices containing 1 UC-thick or 2 UC-thick LCO layers, and in thick (bulk-like) single-phase LCO film. (b) Energy of the magnetic excitations along the $\left(Q_{\|}, 0\right)$ line showing no change as a function of doping. (c) Integrated RIX intensity over the measured reciprocal space showing again no variation with doping from $x(S r)=0$ to $x(S r)=0.4$. The color coding is the same as in panel (b). Data reproduced with permission from $[48,49]$.

Figure 17. HT-IS in one layer of FeSe on STO. (a) Schematic of the fourprobe transport configuration. (b) Temperature dependence of the resistance obtained from linear fits to the I-V characteristics (from [51]). 


\section{REFERENCES:}

[1] J. He, R.F. Klee, G. Logvenov, I. Bozovic, Y. Zhu, Microstructure and possible strain relaxation mechanisms of $\mathrm{La}_{2} \mathrm{CuO}_{4+\delta}$ thin films grown on $\mathrm{LaSrAlO}_{4}$ and $\mathrm{SrTiO}_{3}$ substrates, J. Appl. Phys. 101, 073906 (2007); F. Zheng, G. Logvenov, I. Bozovic, Y. Zhu, J. He, Structural origin of enhanced critical temperature in ultrafine multilayers of cuprate superconducting films, Phys. Rev. B 89, 184509 (2014).

[2] V.L. Ginzburg, On interface superconductivity, Phys. Lett. 13, 101 (1964).

[3] A. Gozar, G. Logvenov, L.F. Kourkoutis, A.T. Bollinger, L.A. Gianuzzi, D.A. Muller, I. Bozovic, High-temperature interface superconductivity between metallic and insulating copper oxides, Nature 455, 782 (2008).

[4] J. Pereiro, A.T. Bollinger, G. Logvenov, A. Gozar, C. Panagopoulos, I. Bozovic, Insights from the study of high-temperature interface superconductivity, Phil. Trans. R. Soc. A 370, 4890 (2012) and references therein.

[5] A. Ohtomo, H.Y. Hwang, A high mobility electron gas at the $\mathrm{LaAlO}_{3} / \mathrm{SrTiO} 3$ heterointerface, Nature 427, 423 (2004).

[6] S. Gariglio, M. Gabay, J. Manhnhart, J.-M. Triscone, Interface superconductivity, Physica C 514, 189 (2015) and references therein.

[7] I. Bozovic, J.N. Eckstein, G.F. Virshup, A. Chaiken, M. Wall, R. Howell, M. Fluss, Atomic-layer engineering of cuprate superconductors, J. Supercond. 7, 187 (1994).

[8] I. Bozovic, Atomic-layer engineering of superconducting oxides: Yesterday, Today, Tomorrow, IEEE Trans. Appl. Supercond. 11, 2686 (2001).

[9] G. Dubuis, X. He, I. Bozovic, Sub-milikelvin stabilization of a closed cycle cryocooler, Rev. Sci. Instr. 85103902 (2014).

[10] G. Logvenov, A. Gozar, I. Bozovic, High temperature interface superconductivity, J. Supercond. Nov. Magn. 26, 2863 (2013).

[11] J.G. Bednorz, K.A. Muller, Possible high-Tc superconductivity in the Ba-La-Cu-O system, Z. Phys. B - Cond. Matter 64, 189 (1986). 
[12] I. Bozovic, G. Logvenov, I. Belca, B. Narimbetov, I. Sveklo, Epitaxial strain and superconductivity in $\mathrm{La} 2-\mathrm{xSrxCuO} 4$ thin films, Phys. Rev. Lett. 89, 107001 (2002).

[13] G. Logvenov, A. Gozar, I. Bozovic, High-temperature superconductivity in a single copper-oxygen plane, Science 326, 699 (2009).

[14] J.W. Loram, K.A. Mirza, P.F. Freeman, The electronic specific heat of $\mathrm{YBa}_{2}\left(\mathrm{Cu}_{1-\mathrm{x}} \mathrm{Zn}_{\mathrm{x}}\right)_{3} \mathrm{O}_{7}$ from $1.6 \mathrm{~K}$ to $300 \mathrm{~K}$.

[15] J.L. Tallon, C. Bernhard, G.V.M. Williams, J.V. Loram, Zn-induced Tc reduction in high- $\mathrm{T}_{\mathrm{c}}$ superconductors: scattering in the presence of a pseudogap, Phys. Rev. Lett. 79, 5294 (1997).

[16] I. Bozovic, G. Logvenov, M.A.J. Verhoeven, P. Caputo, E. Goldobin, T.H. Geballe, No mixing of superconductivity and antiferromagnetism in a high-temperature superconductor, Nature 422, 873 (2003).

[17] A. Gozar, G. Logvenov, V.Y. Butko, I. Bozovic, Surface structure analysis of atomically smooth BaBiO3 films, Phys. Rev. B 75, 201402(R) (2007).

[18] H. Eisaki, N. Kaneto, D.L. Feng, A. Damascelli, P.K. Mang, K.M. Shen, Z.-X. Shen, M. Greven, Effect of chemical inhomogeneity in bismuthbased copper oxide superconductors, Phys. Rev. B 69, 064512 (2004) and references therein.

[19] S.A. Kivelson, Making high- $\mathrm{T}_{\mathrm{c}}$ higher: a theoretical proposal, Physica B 318, 61 (2002).

[20] E. Berg, D. Orgad, S.A. Kivelson, Route to high-temperature superconductivity in composite systems, Phys. Rev. B 78, 094509 (2008).

[21] S. Smadici, J.C.T. Lee, S. Wang, P. Abbamonte, G. Logvenov, A. Gozar, C. Deville Cavellin, I. Bozovic, Superconducting transition at $38 \mathrm{~K}$ in insulating-overdoped $\mathrm{La}_{2} \mathrm{CuO}_{4}-\mathrm{La}_{1.64} \mathrm{Sr}_{0.36} \mathrm{CuO}_{4}$ superlattices: evidence for interface electronic redistribution from resonant soft X-ray scattering, Phys. Rev. Lett. 102, 107004 (2009).

[22] H. Zhou, Y. Yacoby, V.Y. Butko, G. Logvenov, I. Bozovic, R. Pindak, Anomalous expansion of the copper-apical-oxygen distance in superconducting cuprate bilayers, Proc. Natl. Acad. Sci. USA 107, 8103 (2010). 
[23] V.Y. Butko, G. Logvenov, N. Bozovic, Z. Radovic, I, Bozovic, Madelung strain in cuprate superconductors - A route to enhancement of the critical temperature, Adv. Mat. 21, 3644 (2009).

[24] R.E. Glover, M.D. Sherrill, Changes in superconducting critical temperature produced by electrostatic charging, Phys. Rev. Lett., 5, 248 (1960).

[25] C.H. Ahn, S. Gariglio, P. Paruch, T. Tybell, L. Antognazza, J.-M. Triscone, Electrostatic modulation of superconductivity in ultrathin $\mathrm{GdBa}_{2} \mathrm{Cu}_{3} \mathrm{O}_{7-\mathrm{x}}$, Science 284, 1152 (1999).

[26] S. Gariglio, C.H. Ahn, D. Matthey, J.-M. Triscone, Electrostatic tuning of the hole density in $\mathrm{NdBa}_{2} \mathrm{Cu}_{3} \mathrm{O}_{7-\delta}$ films and its effect on the Hall response, Phys. Rev. Lett., 88, 067002 (2002).

[27] S.R. Peck, L.S. Curtin, J.T. McDevitt, R.W. Murray, J.P. Coleman, W.A. Little, T. Zetterer, H.M. Duan, C. Dong, A.M. Herman, Response of the double layer capacitance of a high-temperature superconductor/fluid electrolyte interface to the onset of superconductivity, J. Am. Chem. Soc. 114, 6771 (1992).

[28] S.G. Haupt, D.R. Riley, J.T. McDevitt, Conductive polymer/hightemperature superconductor composite structures, Adv. Mat. 5, 755 (1993).

[29] S.G. Haupt, D.R. Riley, J. Zhao, J.-P. Zhou, J.S. Grassi, J.T. McDevitt, Reversible modulation of superconductivity in $\mathrm{YBa} 2 \mathrm{Cu} 3 \mathrm{O} 7-\delta /$ polypyrrole sandwich structures, Proc. SPIE 2158, 238 (1994).

[30] S.G. Haupt, D.R. Riley, J.S. Grassi, R.-K. Lo, J. Zhao, J.-P. Zhou, J.T. McDevitt, Preparation and characterization of $\mathrm{YBa} 2 \mathrm{Cu} 3 \mathrm{O} 7-\delta /$ polypyrrole bilayer structures, J. Am. Chem. Soc. 116, 9979 (1994).

[31] M.B. Clevenger, C.E. Jones, S.G. Haupt, J Zhao, J.T. McDevitt, Organic conductor / high-Tc superconductor bilayer structures, Proc. SPIE 2697, 508 (1996).

[32] H. Yuan, H. Shimotani, A. Tsukazaki, A. Ohtomo, M. Kawasaki, Y. Iwasa, High-density carrier accumulation in $\mathrm{ZnO}$ field-effect transistors gated by electric double layers of ionic liquids, Adv. Mat. 19, 1046 (2009). 
[33] H. Shimotani, H. Suzuki, K. Ueno, M. Kawasaki, Y. Iwasa, p-type field-effect transistor of $\mathrm{NiO}$ with electric double-layer gating, Appl. Phys. Lett. 92, 242107 (2008).

[34] K. Ueno, S. Nakamura, H. Shimotani, A. Ohtomo, N. Kimura, T. Nojima, H. Aoki, Y. Iwasa, M. Kawasaki, Electric-field-induced superconductivity in an insulator, Nat. Mat. 7, 855 (2008).

[35] K. Ueno, S. Nakamura, H. Shimotani, H.T. Yuan, N. Kimura, T. Nojima, H. Aoki, Y. Iwasa, M. Kawasaki, Discovery of superconductivity in $\mathrm{KTaO}_{3}$ by electrostatic carrier doping, Nat. Nanotechnol. 6, 408 (2011).

[36] J. Jeong, N. Aetukuri, T. Graf, T.D. Schladt, M.G. Samant, S.S.P. Parkin, Suppression of metal-insulator transition in $\mathrm{VO}_{2}$ by electric fieldinduced Oxygen vacancy formation, Science 339, 1402 (2013).

[37] H.T. Yi, B. Gao, W. Xie, S.-W. Cheong, V. Podzorov, Tuning the metal-insulator crossover and magnetism in $\mathrm{SrRuO}_{3}$ by ionic gating, Sci. Rep. 4, 6604 (2014).

[38] X. Leng, J. Pereiro, J. Strle, G. Dubuis, A.T. Bollinger, A. Gozar, C. Panagopoulos, D. Pavuna, I. Bozovic, Insulator to metal transition in WO3 induced by electrolyte gating, submitted.

[39] A.T. Bollinger, G. Dubuis, J. Yoon, D. Pavuna, J. Misewich, I. Bozovic, Superconductor-insulator transition in $\mathrm{La}_{2-\mathrm{x}} \mathrm{Sr}_{\mathrm{x}} \mathrm{CuO}_{4}$ at the pair quantum resistance, Nature 472, 458 (2011).

[40] X. Leng, J. Garcia-Barriocanal, S. Bose, Y. Lee, A.M. Goldman, Electrostatic control of the evolution from a superconducting state to an insulating phase in ultrathin $\mathrm{YBa}_{2} \mathrm{Cu}_{3} \mathrm{O}_{7-\delta}$ films, Phys. Rev. Lett. 107, 027001 (2011).

[41] A.M. Goldman, Superconductor-insulator transitions, Int. J. Mod. Phys. B 24, 4801 (2010).

[42] Y.-H. Lin, J. Nelson, A.M. Goldman, Superconductivity of very thin films: the superconductor-insulator transition, Physica C 514, 130 (2015).

[43] Y. Yacoby, H. Zhou, R. Pindak, I. Bozovic, Atomic-layer synthesis and imaging uncover broken inversion symmetry in $\mathrm{La}_{2-x} \mathrm{Sr}_{x} \mathrm{CuO}_{4}$ films, Phys. Rev. B 87, 014108 (2013). 
[44] R.R. Mehta, B.D. Silverman, J.T. Jacobs, Depolarization fields in thin ferroelectric films, J. Appl. Phys. 44, 3379 (1973).

[45] J. Junquera, P. Ghosez, Critical thickness for ferroelectricity in perovskite ultrathin films, Nature 422, 506 (2003).

[46] C.H. Ahn, K.M. Rabe, J.-M. Triscone, Ferroelectricity at the nanoscale: local polarization in oxide thin films and heterostructures, Science 303, 488 (2004) and references therein.

[47] J. Wu, O. Pelleg, A.T. Bollinger, Y.-J. Sun, G.S. Boebinger, M. Vanevic, Z. Radovic, I. Bozovic, Anomalous independence of interface superconductivity from carrier density, Nat. Mater. 12, 877 (2013).

[48] V.A. Gasparov, I. Bozovic, Magnetic field and temperature dependence of complex conductance of ultrathin $\mathrm{La}_{1.55} \mathrm{Sr}_{0.45} \mathrm{CuO}_{4} / \mathrm{La}_{2} \mathrm{CuO}_{4}$.

[49] M.P.M. Dean, R.S. Springell, C. Mooney, K.J. Zhou, J. Pereiro, I. Bozovic, B. Dalla Piazza, H.M. Ronnow, E. Morenzoni, J. van der Brink, T. Schmitt, J.P. Hill, Spin excitations in a single $\mathrm{La}_{2} \mathrm{CuO}_{4}$ layer, Nat. Mater. 11, 850 (2012).

[50] M.P.M. Dean, G. Dellea, R.S. Springell, F. Yakhou-Harris, K. Kummer, N.B. Brookes, X. Liu, Y.-J. Sun, J. Strle, T. Schmitt, L. Braicovich, G. Ghiringhelli, I. Bozovic, J.P. Hill, Persistence of magnetic excitations in $\mathrm{La}_{2-\mathrm{x}} \mathrm{Sr}_{\mathrm{x}} \mathrm{CuO}_{4}$ from the undoped insulator to the heavily overdoped non-superconducting metal, Nat. Mater. 12, 1019 (2013).

[51] J.-F. Ge, Z.-L. Liu, C. Liu, C.-L. Gao, D. Qian, Q.-K. Xue, Y. Liu, J.-F. Jia, Superconductivity above $100 \mathrm{~K}$ in a single layer FeSe films on doped $\mathrm{SrTiO}_{3}$, Nat. Mater. 14, 285 (2014).

[52] I. Bozovic and C.H. Ahn, A new frontier for superconductivity, Nat. Phys. 10, 892 (2014) and references therein. 
Fig. 1

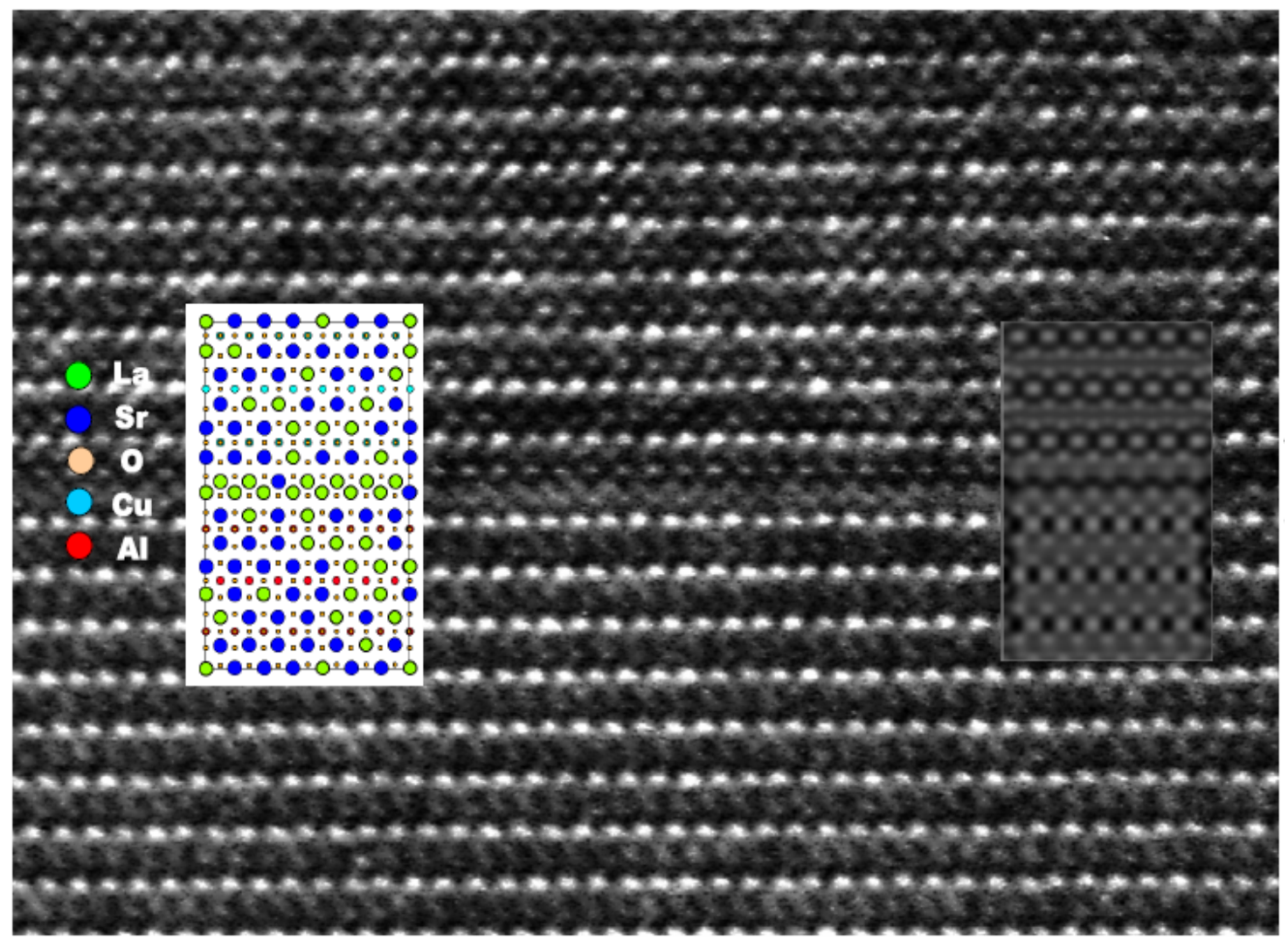


Fig. 2

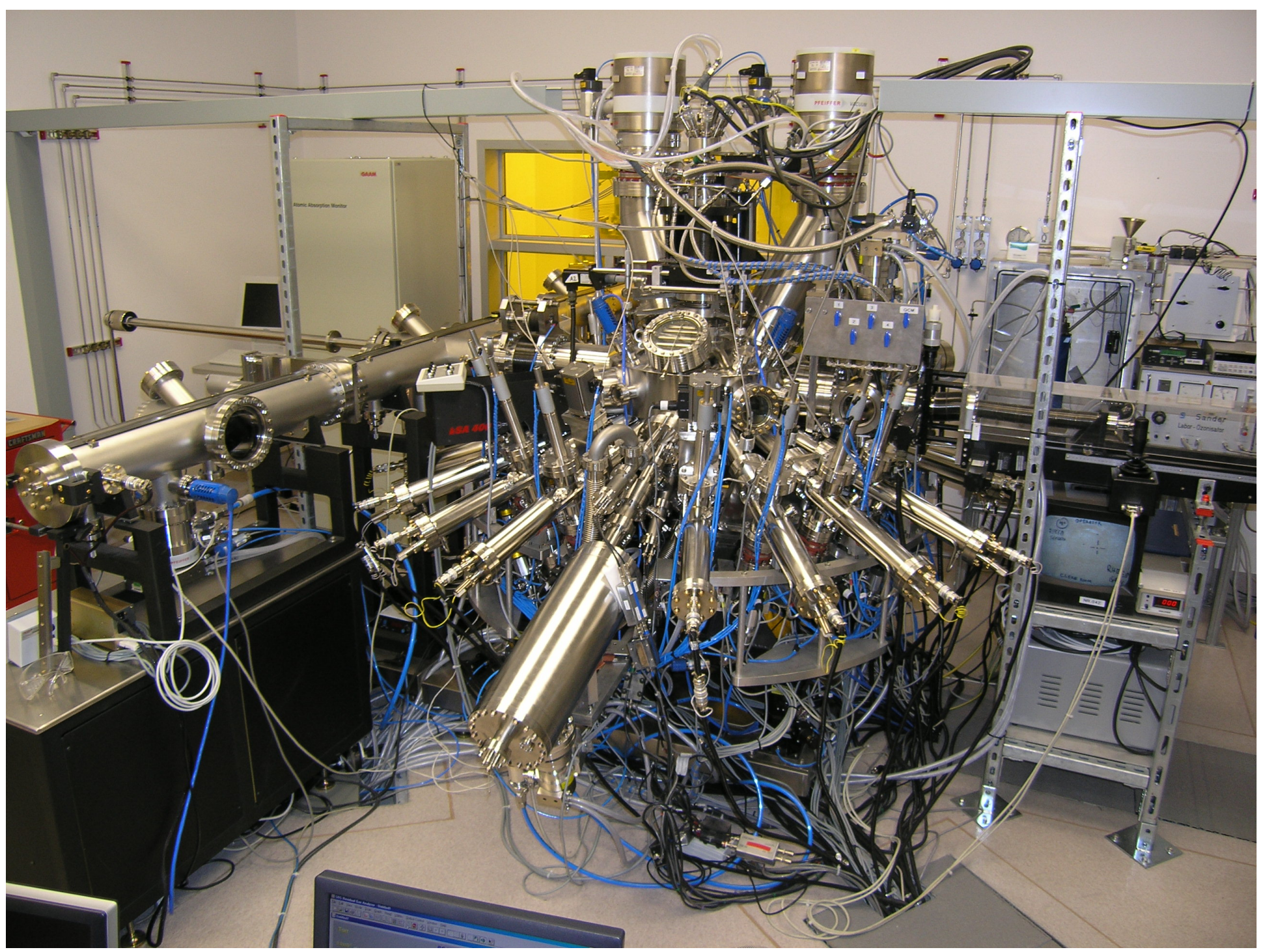


Fig. 3

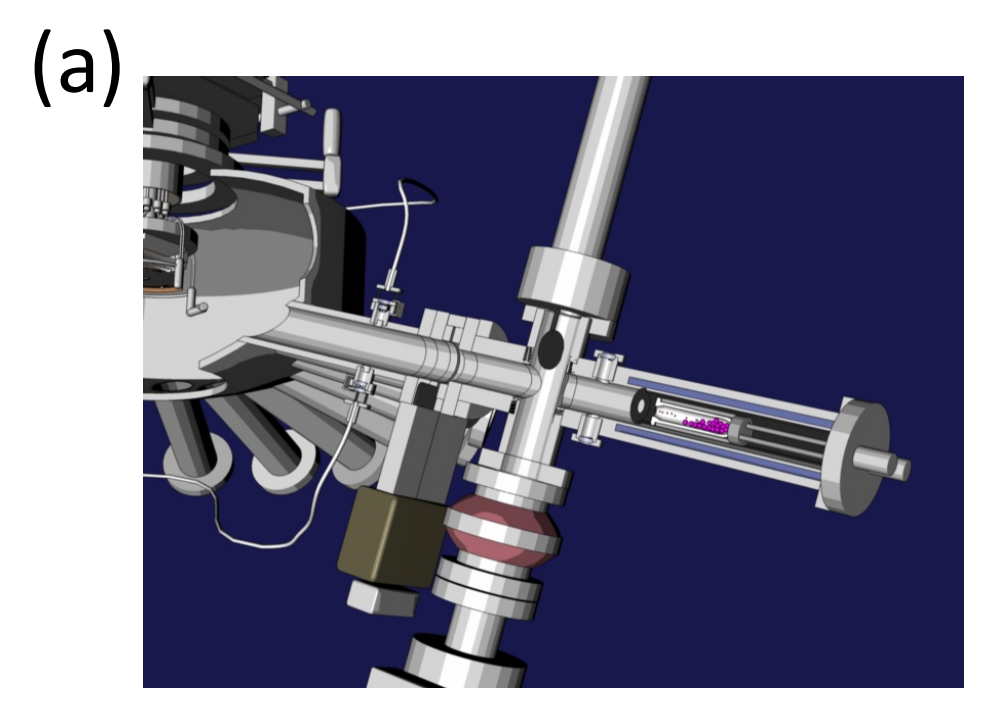

(b)

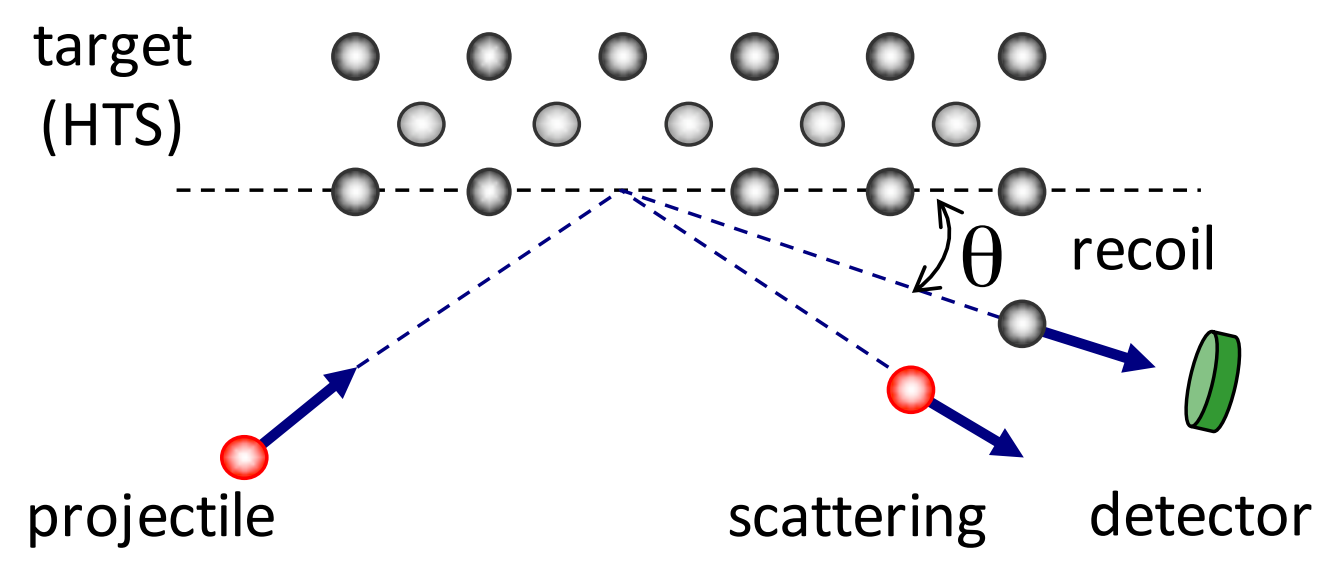

(c)
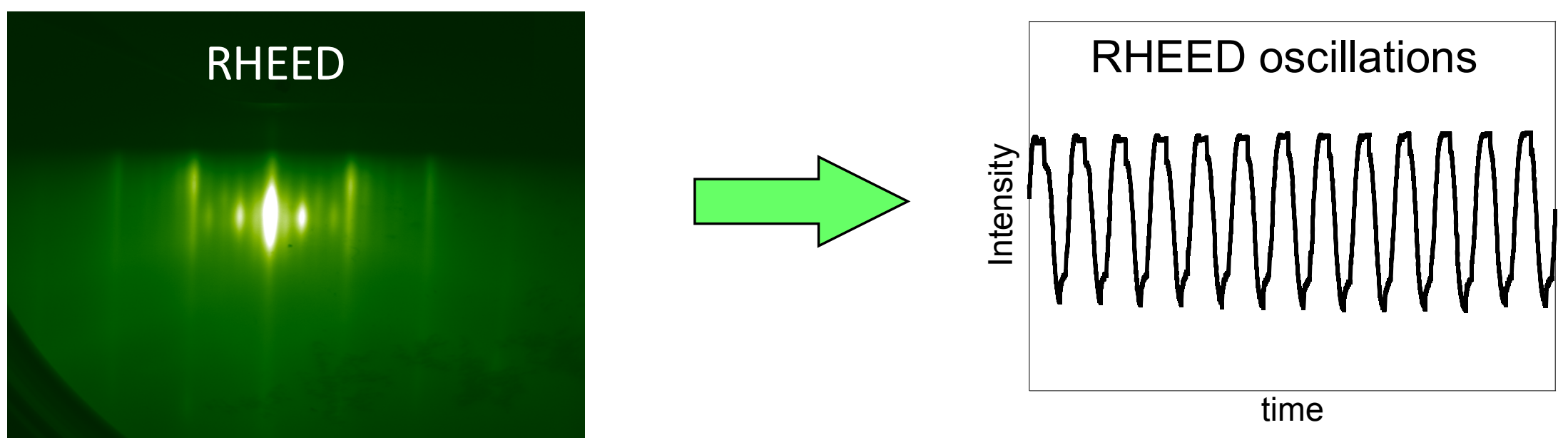
Fig. 4
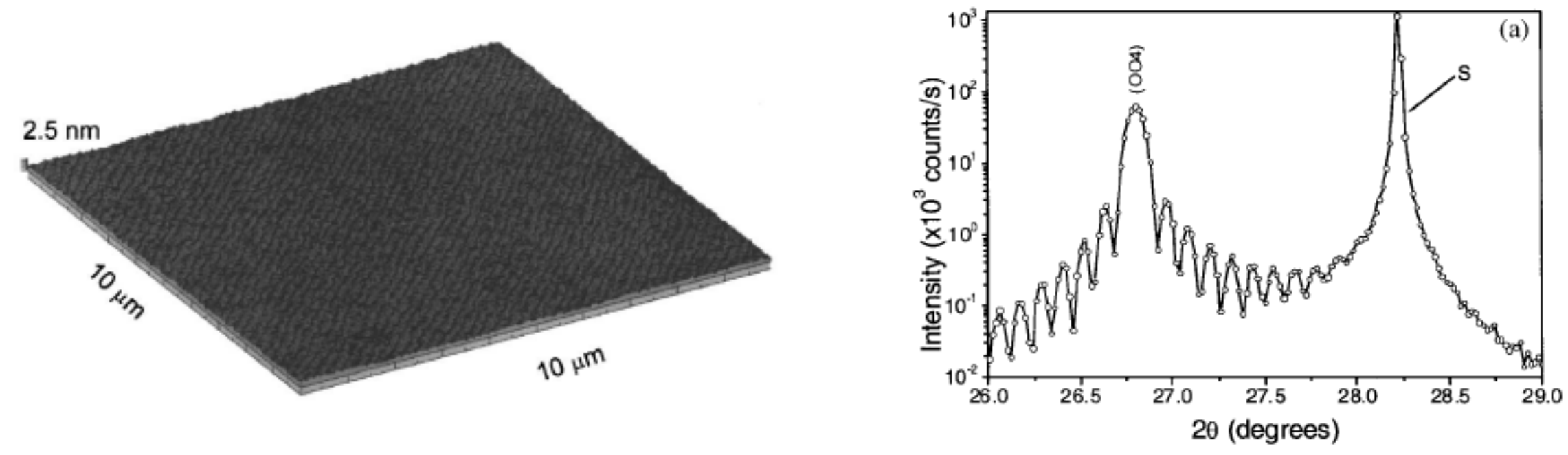


\section{Fig. 5}

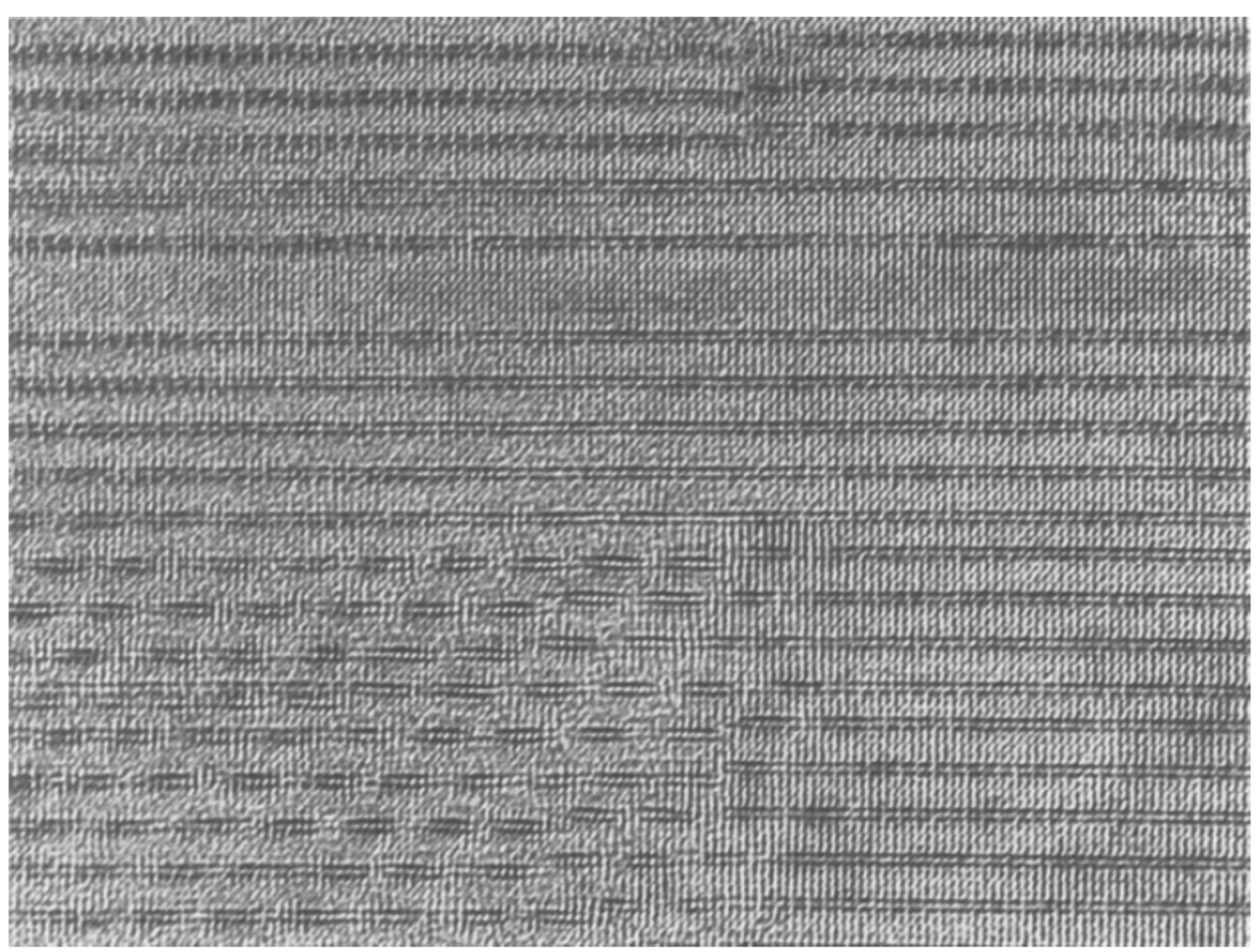


Fig. 6
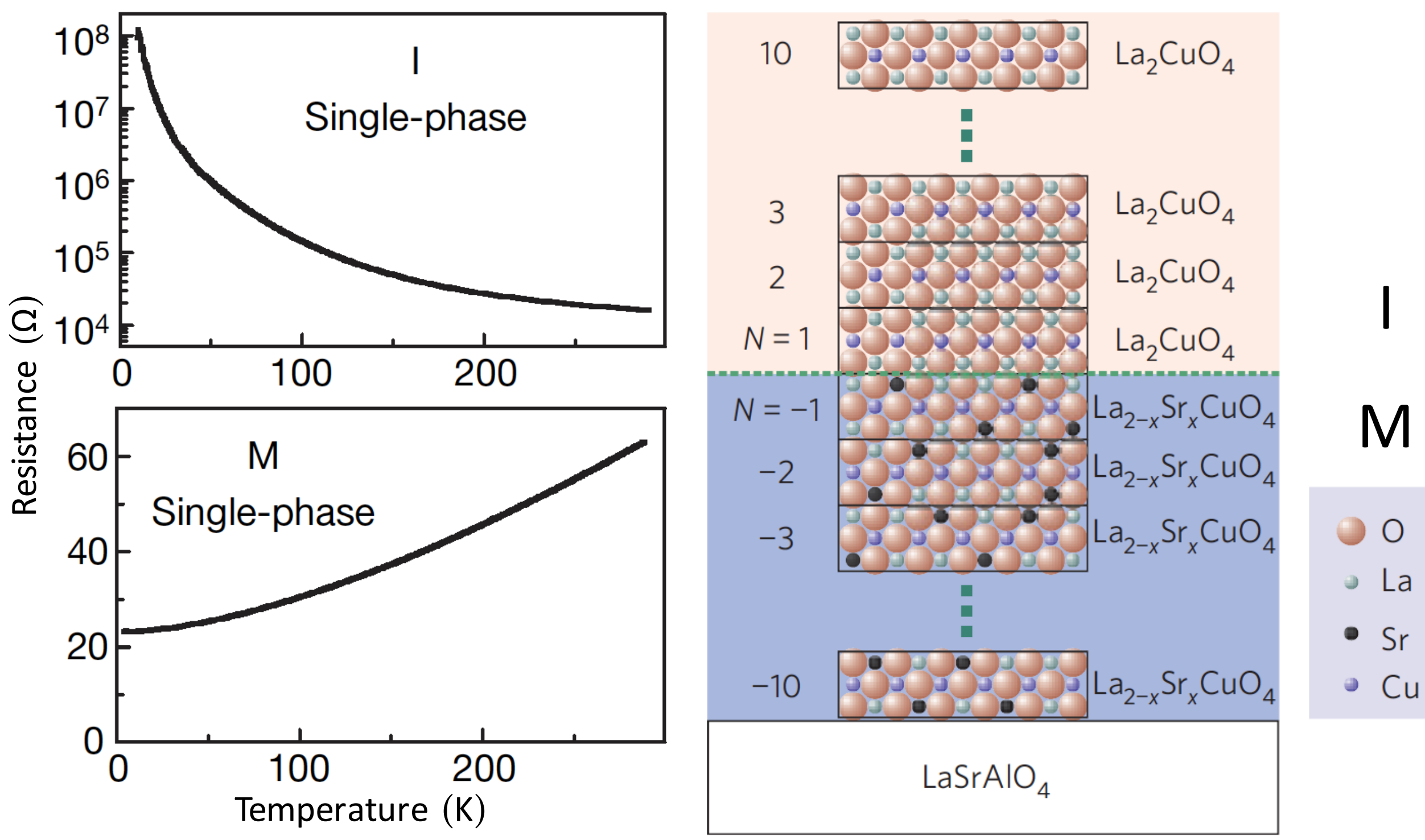
Fig. 7

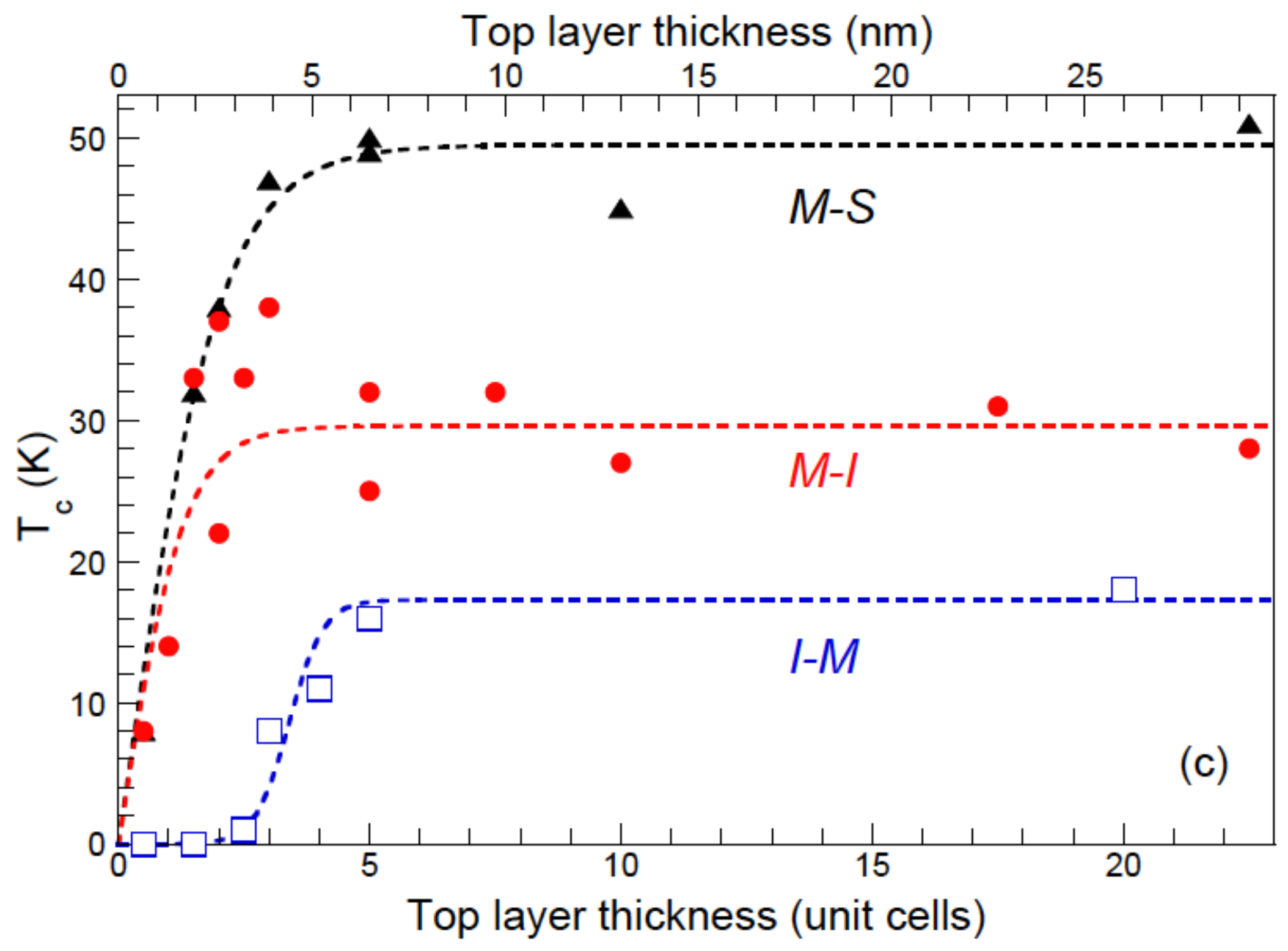


Fig. 8

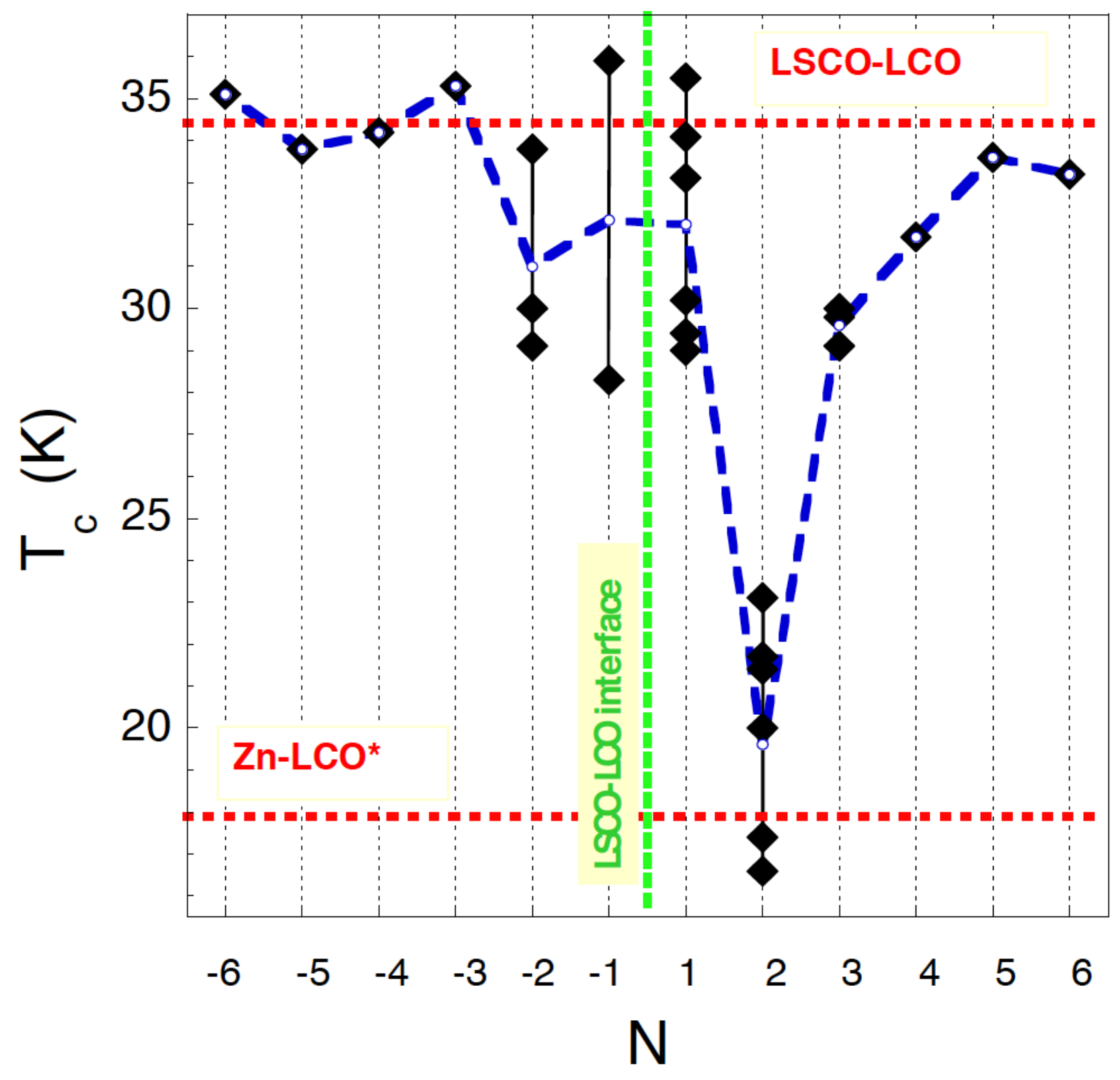


Fig. 9
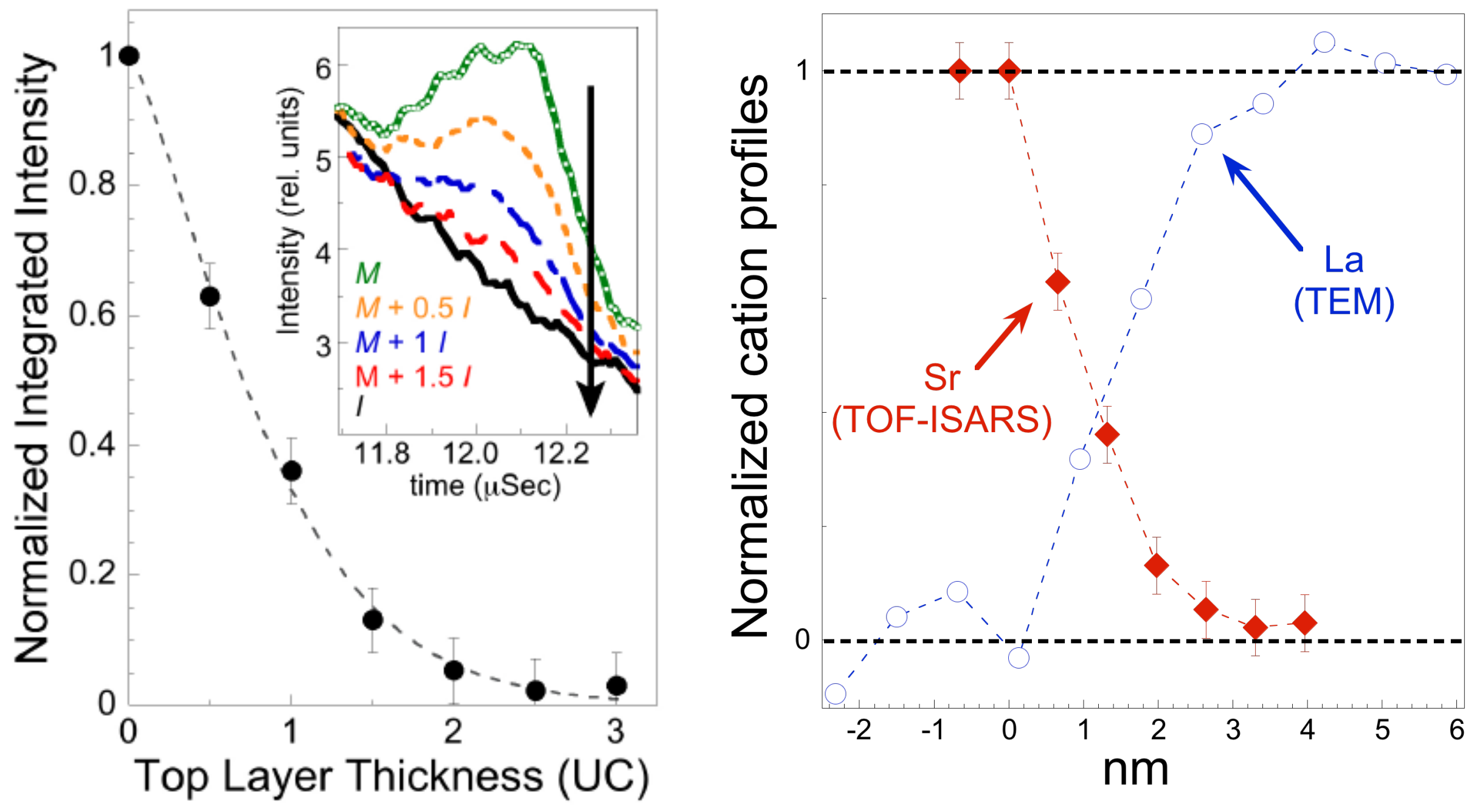
Fig. 10

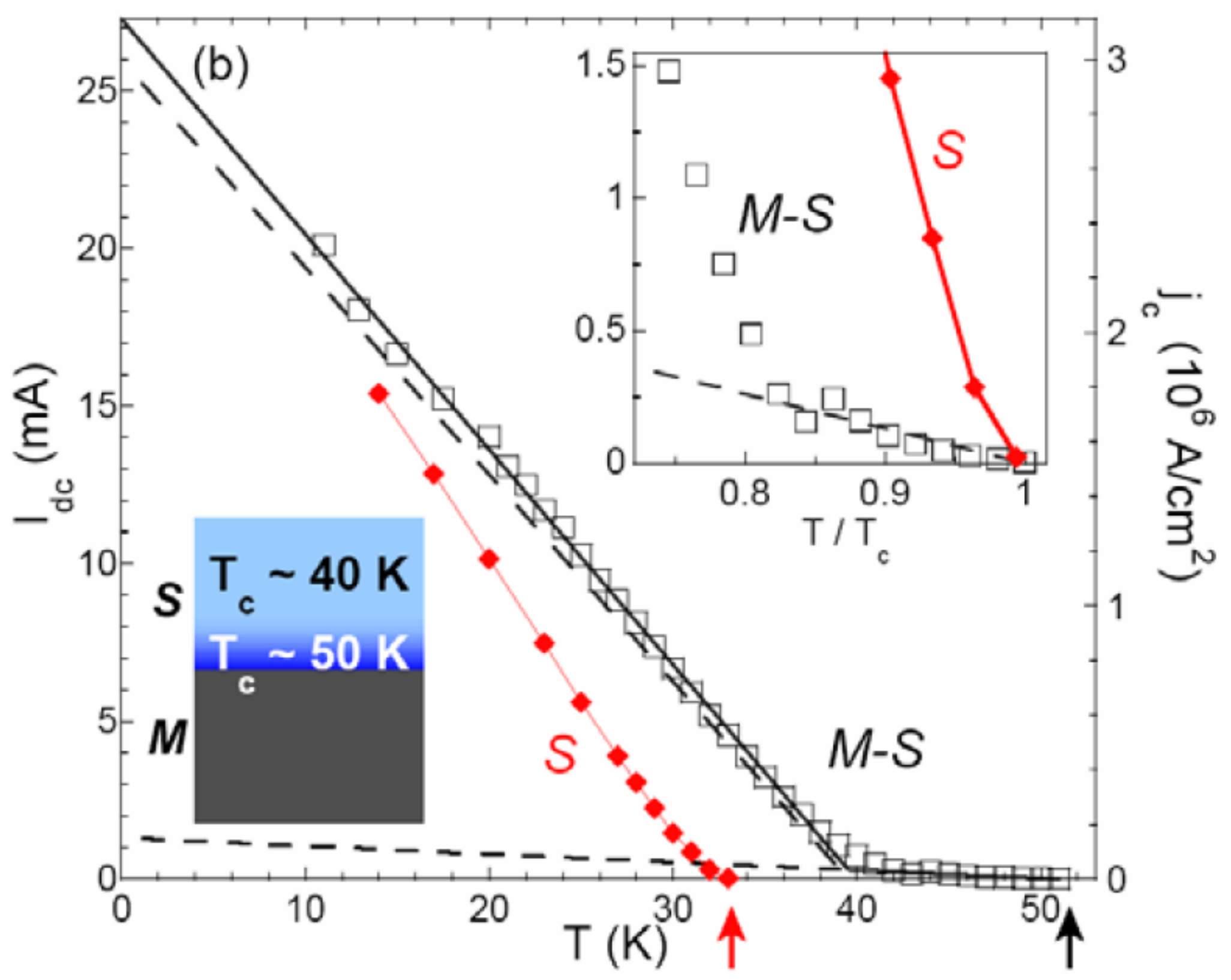


Fig. 11

(a)

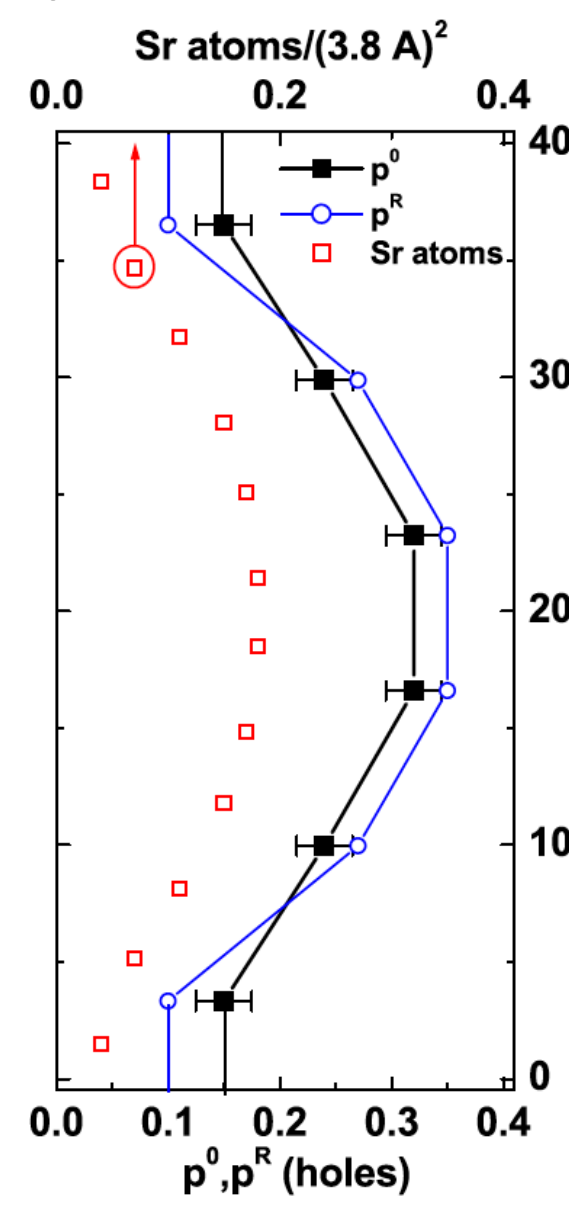

(b)

\begin{tabular}{|c|c|}
\hline \multirow{3}{*}{$\mathrm{p}_{\min }$} & $\mathrm{CuO}_{2}$ \\
\hline & $\mathrm{La}_{0.96} \mathrm{Sr}_{0.04} \mathrm{O}$ \\
\hline & $\mathrm{La}_{0.96} \mathrm{Sr}_{0.04} \mathrm{O}$ \\
\hline \multirow[t]{3}{*}{$\mathrm{p}_{\text {min }}$} & $\mathrm{CuO}_{2}$ \\
\hline & $\mathrm{La}_{0.93} \mathrm{Sr}_{0.07} \mathrm{O}$ \\
\hline & $\mathrm{La}_{0.89} \mathrm{Sr}_{0.11} \mathrm{O}$ \\
\hline \multirow[t]{3}{*}{$\mathrm{p}_{\text {int }}$} & $\mathrm{CuO}_{2}$ \\
\hline & $\mathrm{La}_{0.85} \mathrm{Sr}_{0.15} \mathrm{O}$ \\
\hline & $\mathrm{La}_{0.83} \mathrm{Sr}_{0.17} \mathrm{O}$ \\
\hline \multirow[t]{3}{*}{$\mathrm{p}_{\max }$} & $\mathrm{CuO}_{2}$ \\
\hline & $\mathrm{La}_{0.82} \mathrm{Sr}_{0.18} \mathrm{O}$ \\
\hline & $\mathrm{La}_{0.82} \mathrm{Sr}_{0.18} \mathrm{O}$ \\
\hline \multirow[t]{3}{*}{$\mathrm{p}_{\text {max }}$} & $\mathrm{CuO}_{2}$ \\
\hline & $\mathrm{La}_{0.83} \mathrm{Sr}_{0.17} \mathrm{O}$ \\
\hline & $\mathrm{La}_{0.85} \mathrm{Sr}_{0.15} \mathrm{O}$ \\
\hline \multirow[t]{3}{*}{$\mathrm{p}_{\text {int }}$} & $\mathrm{CuO}_{2}$ \\
\hline & $\mathrm{La}_{0.89} \mathrm{Sr}_{0.11} \mathrm{O}$ \\
\hline & $\mathrm{La}_{0.93} \mathrm{Sr}_{0.07} \mathrm{O}$ \\
\hline \multirow[t]{3}{*}{$\mathrm{p}_{\min }$} & $\mathrm{CuO}_{2}$ \\
\hline & $\mathrm{La}_{0.96} \mathrm{Sr}_{0.04} \mathrm{O}$ \\
\hline & $\mathrm{La}_{0.96} \mathrm{Sr}_{0.04} \mathrm{O}$ \\
\hline $\mathrm{p}_{\min }$ & $\mathrm{CuO}_{2}$ \\
\hline
\end{tabular}

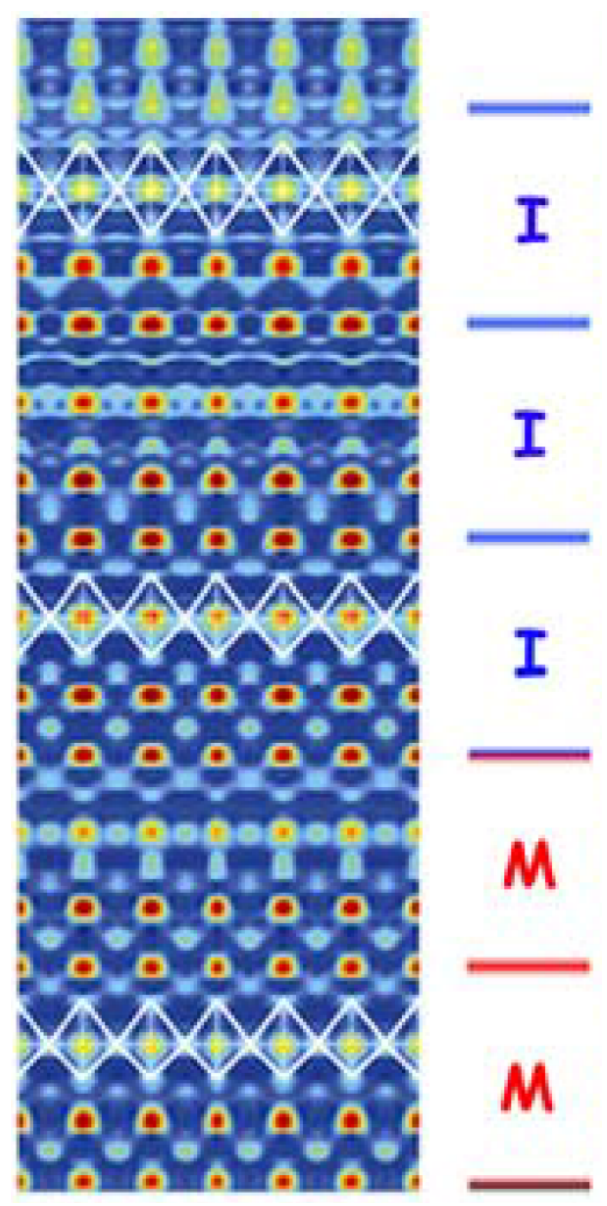


Fig. 12

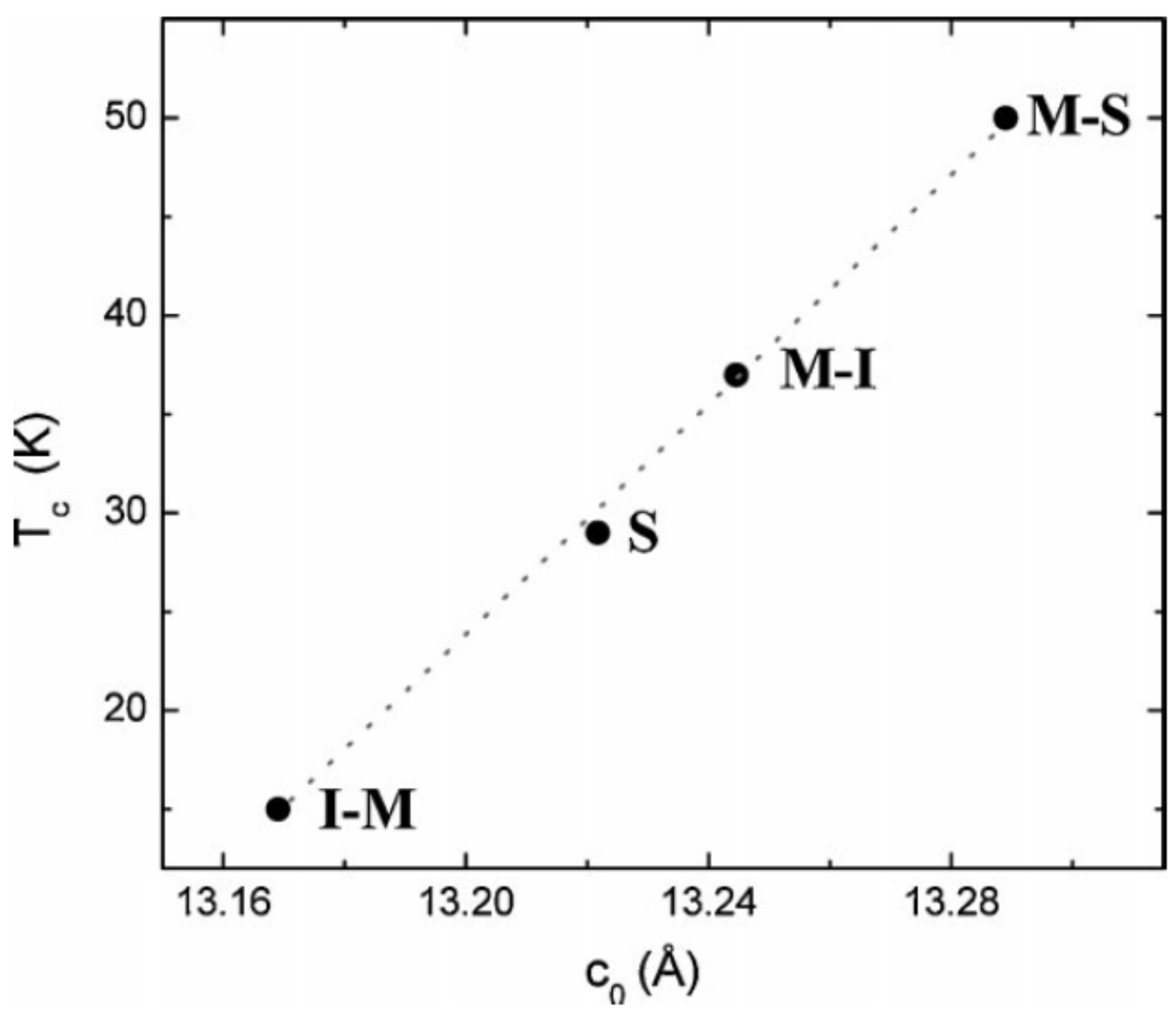


Fig. 13
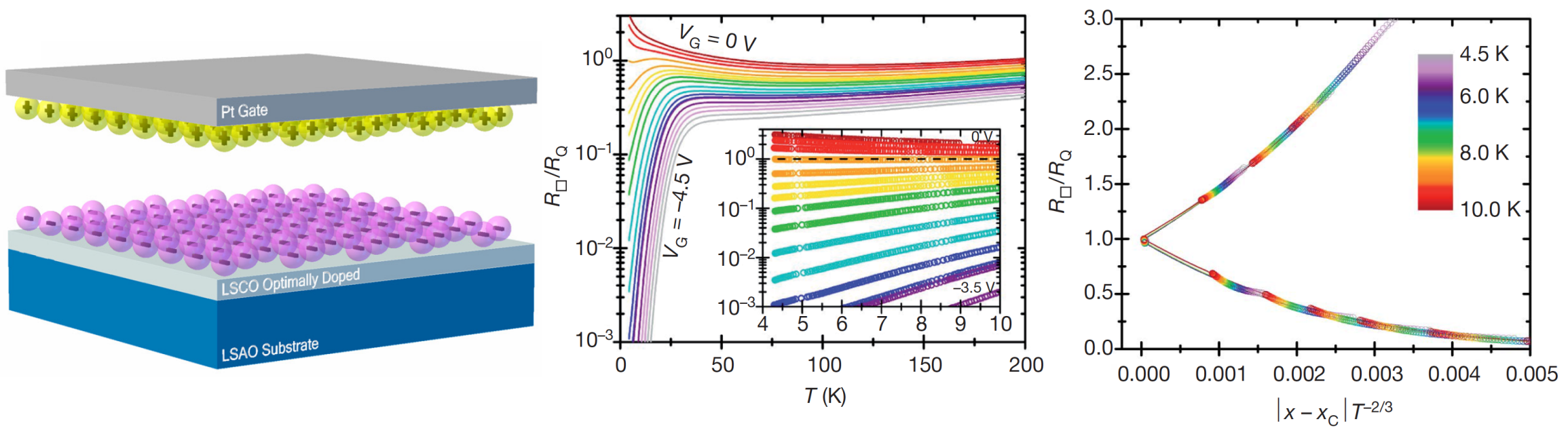
Fig. 14

(a)
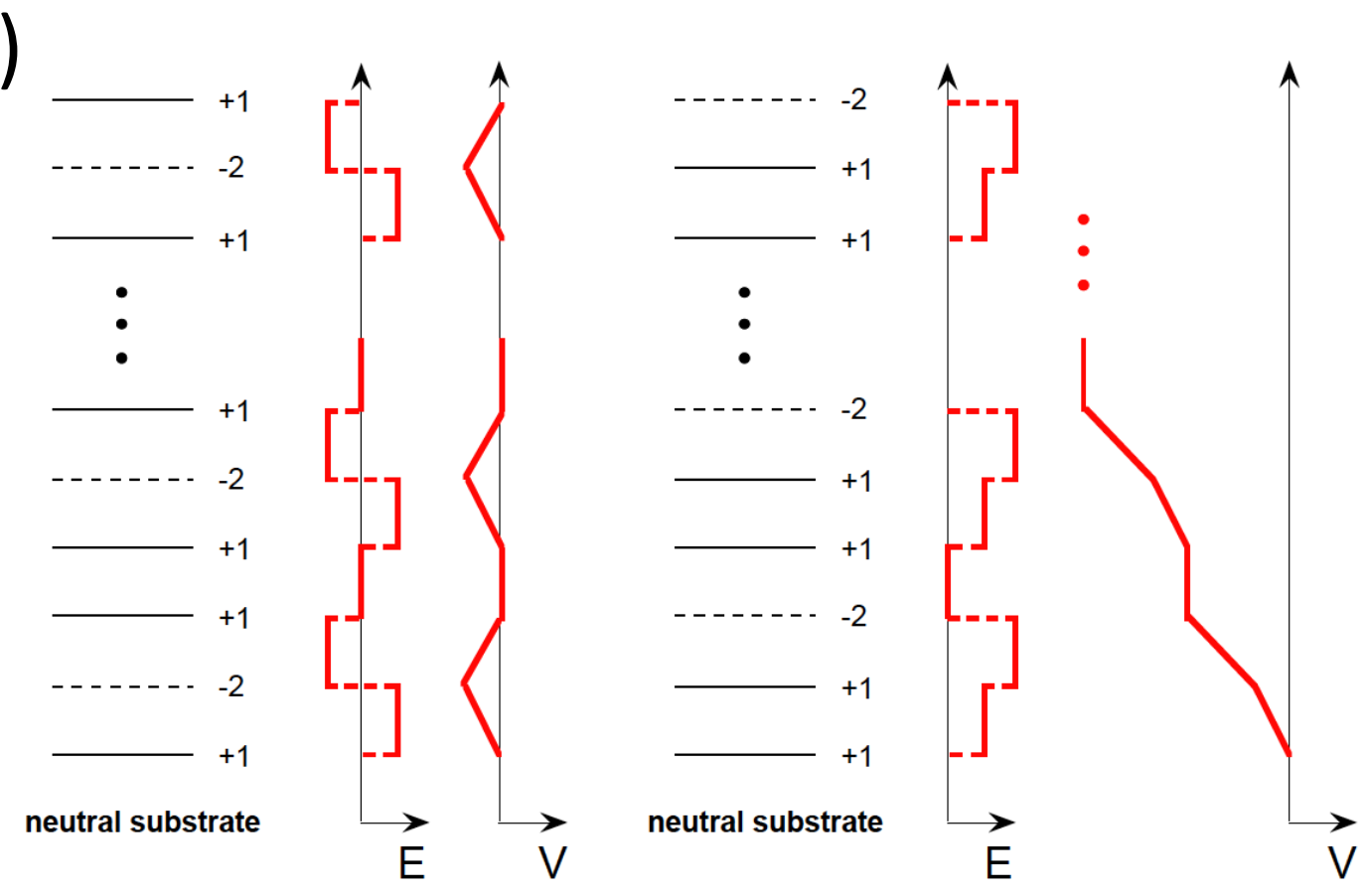

(b)

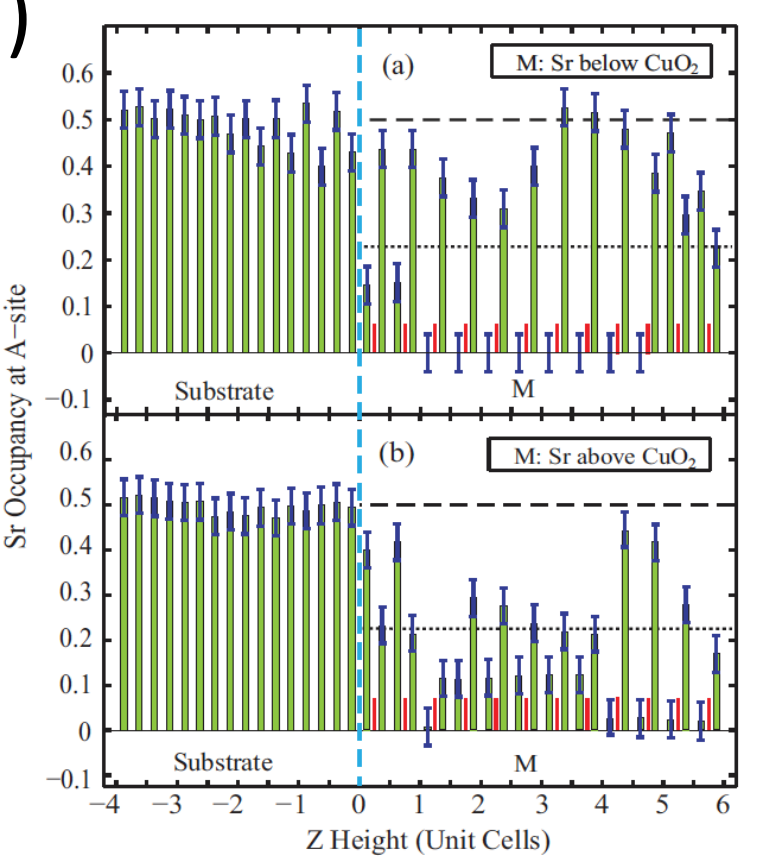


Fig. 15

(a)

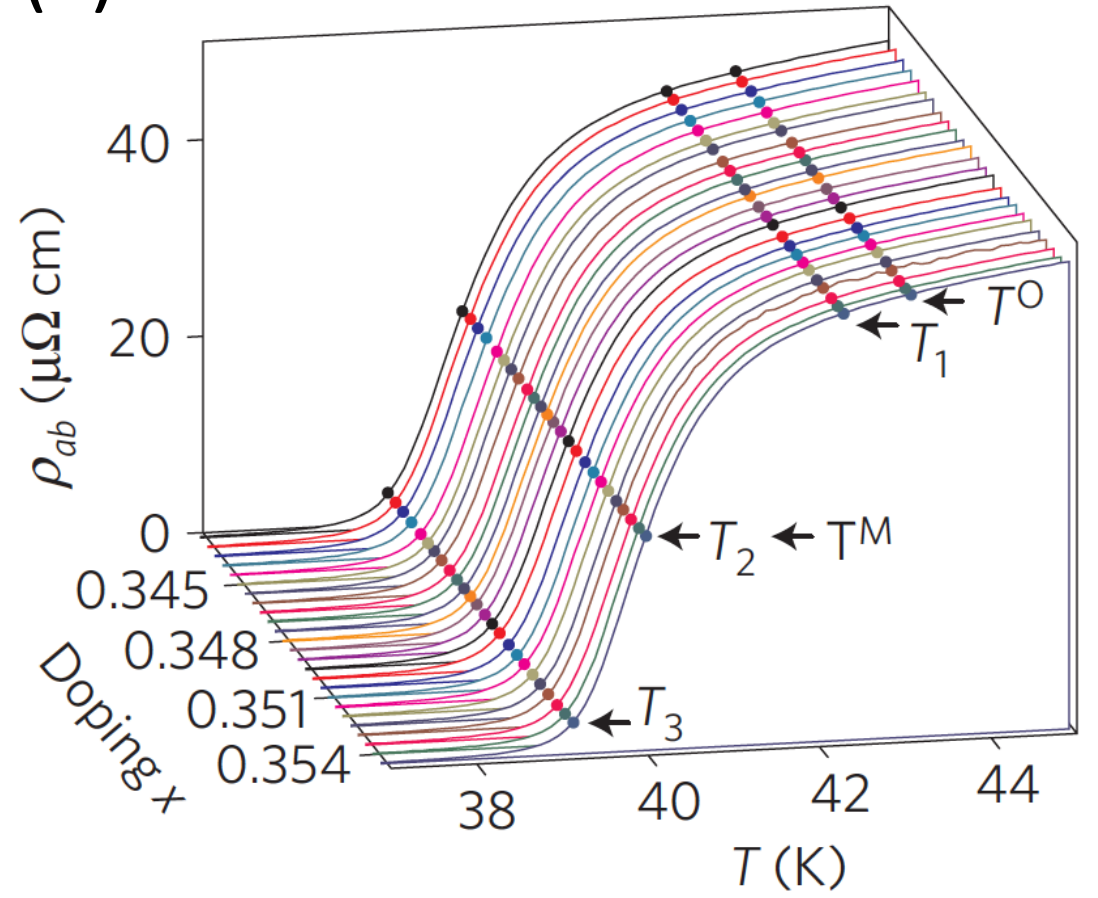

(b)

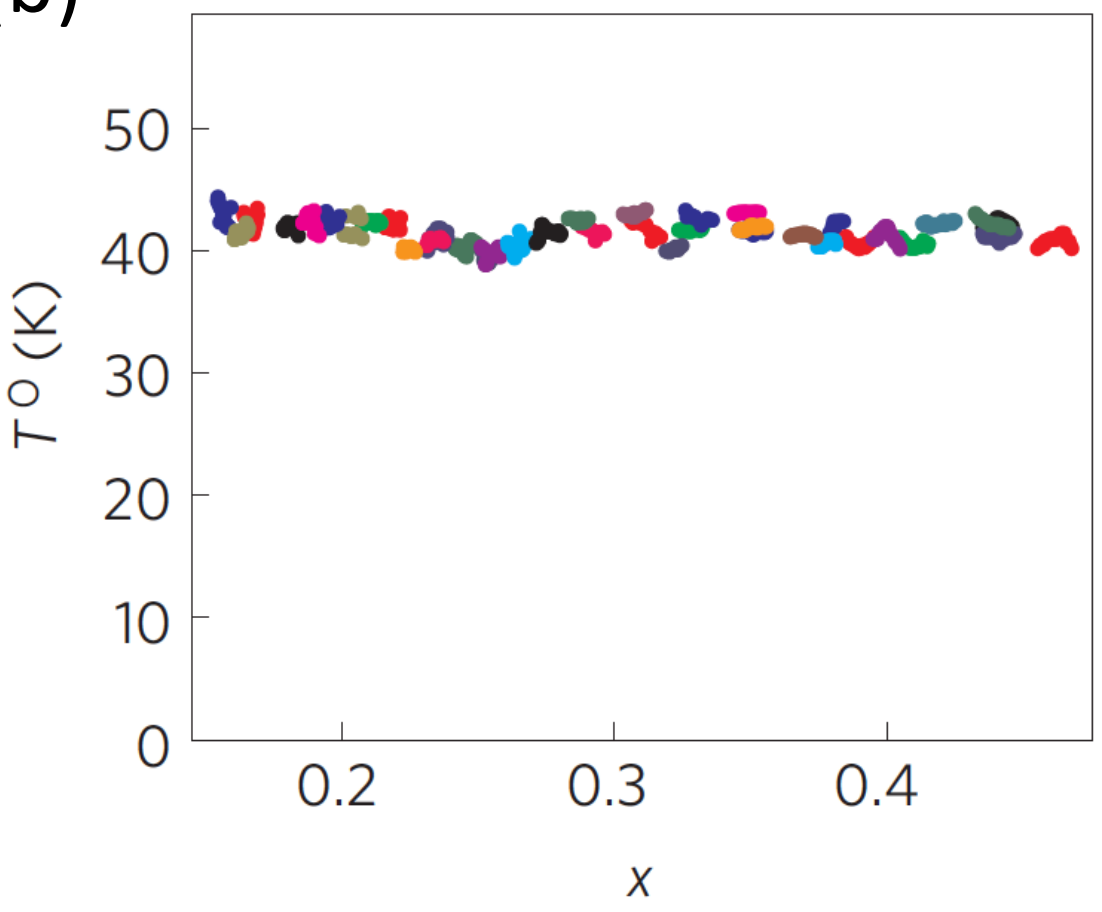


Fig. 16

(a)

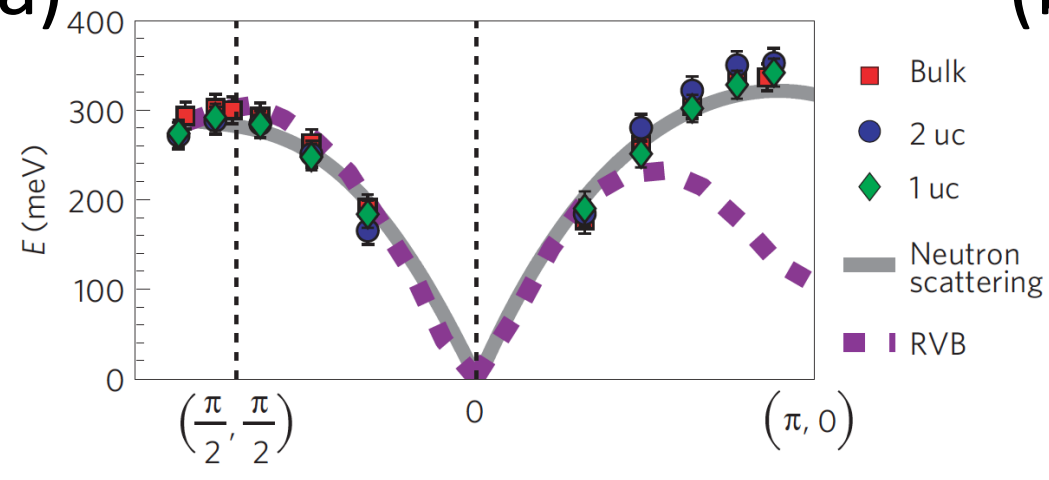

(b)

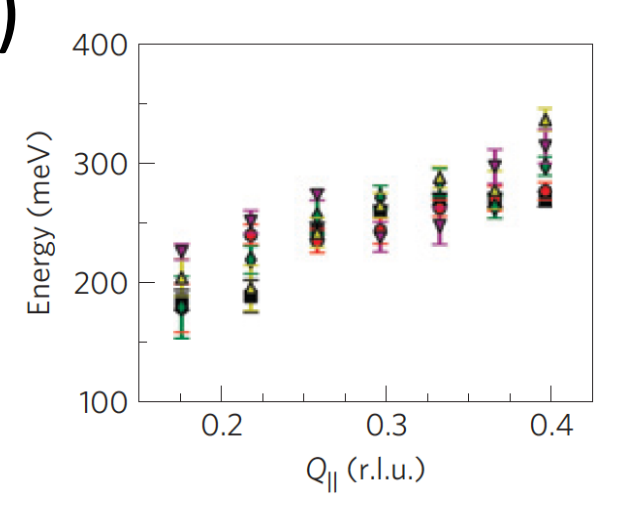

(c)

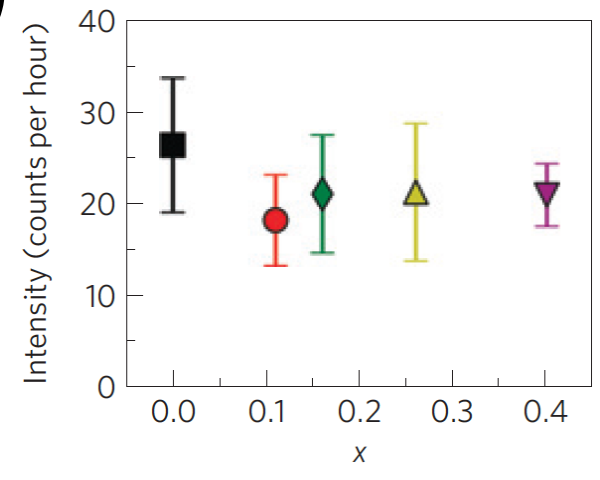


Fig. 17

(a)

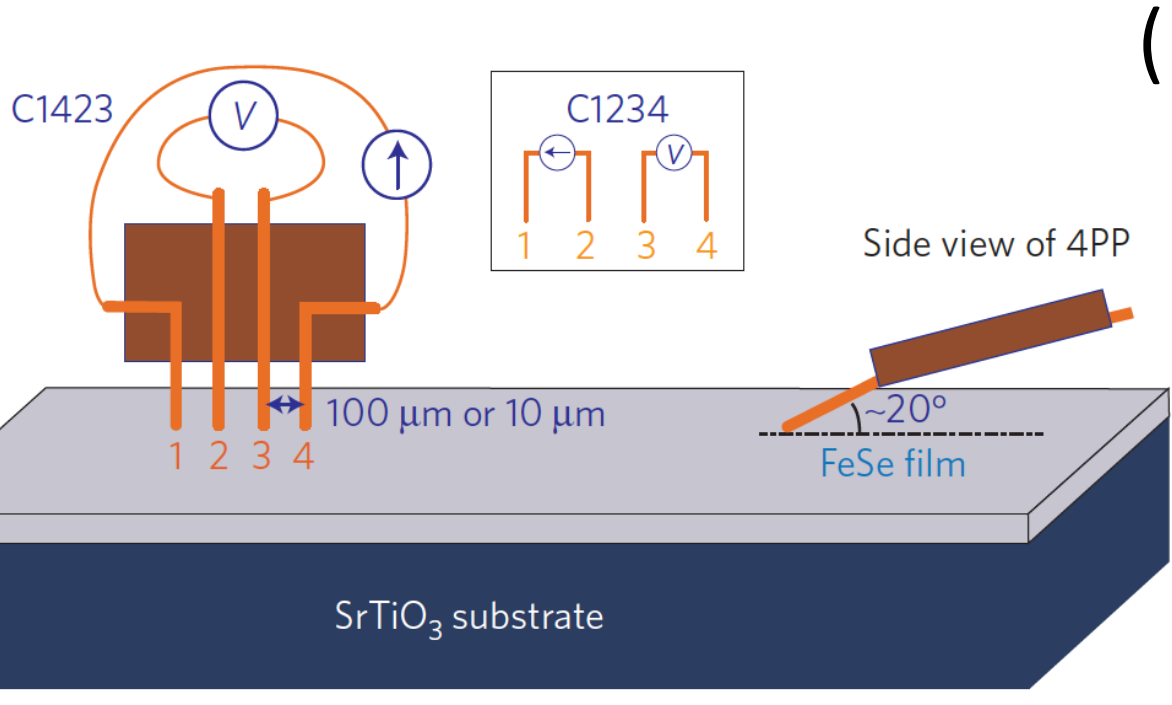

(b)

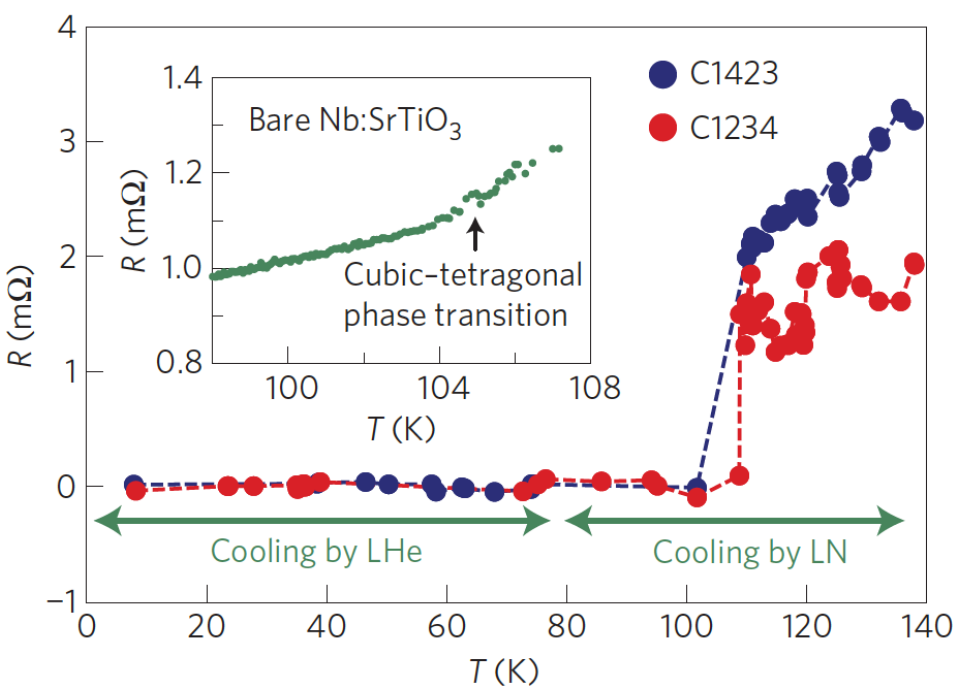

